\title{
Resistance to Second-Line Anti-TB Drugs in Cambodia: A Phenotypic and Genetic Study
}

This article was published in the following Dove Press journal:

Infection and Drug Resistance

Sokleaph Cheng ${ }^{1-3}$
Mallorie Hide ${ }^{3-5}$
Sok Heng Pheng
Alexandra Kerléguer
Gauthier Delvallez
Sophan Sam iD
${ }^{7}$
Tan Eang Mao ${ }^{6}$
Thi Van Anh Nguyen ${ }^{3,8}$
Anne-Laure Bañuls ${ }^{3-5}$
'Institut Pasteur du Cambodge and
Ministry of Health, Phnom Penh,
Cambodia; ${ }^{2}$ Medical Biology Laboratory,
Institut Pasteur du Cambodge, Phnom
Penh, Cambodia; ${ }^{3}$ LMI Drug Resistance in
South East Asia, Institut Pasteur du
Cambodge, Phnom Penh, Cambodia;
${ }^{4}$ MIVEGEC, University of Montpellier,
Institute of Research for Development,
Centre National de la Recherche
Scientifique, Montpellier, France; ${ }^{5}$ CREES
(Centre de Recherche En Écologie Et
Évolution de la Santé), Montpellier,
France; ${ }^{6}$ National Center for
Tuberculosis and Leprosy Control,
Phnom Penh, Cambodia; ${ }^{7}$ Cambodian
Health Committee, Phnom Penh,
Cambodia; ${ }^{8}$ Department of Bacteriology,
National Institute of Hygiene and
Epidemiology, Hanoi, Martinique,
Vietnam
Vietnam
Background: Due to the emergence of Mycobacterium tuberculosis (M.tb) clinical isolates resistant to most potent first-line drugs (FLD), second-line drugs (SLD) are being prescribed more frequently. We explore the genetic characteristics and molecular mechanisms of M.tb isolates phenotypically resistant to SLD, including pre-extensively drug-resistant (pre-XDR) and extensively drug-resistant (XDR) isolates.

Methods: Drug-resistant (DR) M.tb isolates collected from 2012 to 2017 were tested using sequencing and phenotypic drug susceptibility testing. Genotypes were determined to explore their links with SLD resistance patterns.

Results: Of the 272 DR M.tb isolates, 6 non-multidrug resistant (non-MDR) isolates were fluoroquinolones (FQ)-resistant, 3 were XDR and 16 were pre-XDR (14 resistant to FQ and 2 to second-line injectable drugs). The most frequent mutations in FQ-resistant and secondline injectable drugs resistant isolates were gyrA D94G (15/23) and $\operatorname{rrs}$ a1401g (3/5), respectively. Seventy-five percent of pre-XDR isolates and $100 \%$ of XDR isolates harbored mutations conferring resistance to pyrazinamide. All XDR isolates belonged to the Beijing genotype, of which one, named $\mathrm{XDR}+$, was resistant to all drugs tested. One cluster including pre-XDR and XDR isolates was observed.

Conclusion: This is the first description of SLD resistance in Cambodia. The data suggest that the proportion of XDR and pre-XDR isolates remains low but is on the rise compared to previous reports. The characterization of the $\mathrm{XDR}+$ isolate in a patient who refused treatment underlines the risk of transmission in the population. In addition, genotypic results show, as expected, that the Beijing family is the main involved in pre-XDR and XDR isolates and that the spread of the Beijing pre-XDR strain is capable of evolving into XDR strain. This study strongly indicates the need for rapid interventions in terms of diagnostic and treatment to prevent the spread of the pre-XDR and XDR strains and the emergence of more resistant ones.

Keywords: Mycobacterium tuberculosis, extensively drug-resistant tuberculosis, preextensively drug-resistant tuberculosis, fluoroquinolone, second-line injectable drugs

\section{Introduction}

Nowadays, tuberculosis (TB) is the leading cause of death from a single infectious agent, Mycobacterium tuberculosis (M.tb), ranging above HIV. In 2018, an estimated 10.0 million people developed TB and around 1.5 million people died of this disease, including 1.25 million deaths from HIV-negative individuals and 251,000 deaths from HIV-associated TB. Drug-resistant TB (DR-TB) is of major concern to TB control worldwide, as no country or region is spared. The emergence of multidrug-resistant (MDR) (TB-resistant to at least rifampicin and isoniazid) and extensively drug-resistant (XDR) (MDR plus resistance to any fluoroquinolone and
Correspondence: Sokleaph Cheng Institut Pasteur du Cambodge, No. 5 Monivong Boulevard, Phnom Penh 120210, Cambodia

Tel +855 12222684

Email csokleaph@pasteur-kh.org
Infection and Drug Resistance 2021:14 1089-1104

1089 
any second-line injectable drug) M.tb isolates, along with the lack of new effective drugs to fight the disease, is a growing problem. An estimated 484,000 MDR-TB cases emerged in 2018 and among the incident of TB cases, $3.4 \%$ of new cases and $18 \%$ of previously treated cases were estimated to have MDR-TB. As of 2018, XDRTB has been identified in 127 countries, with an average proportion of XDR-TB of $8.5 \%$ among MDR-TB cases. ${ }^{1}$ Totally drug-resistant (TDR) TB has also been described, but the term is not yet recognized by the World Health Organization (WHO). ${ }^{2-4}$

Multiple factors have led to the emergence of XDR M. tb isolates, including underdeveloped laboratory capacities that prevent timely diagnosis of MDR-TB and limited access to second-line drugs to treat MDR-TB. ${ }^{5}$ Evidencebased treatment is considered as the best treatment option to prevent the development of drug-resistant $\mathrm{TB}^{6}$ However, due to the delay of the results, limited access to conventional drug susceptibility testing (DST) and the unreliable results for some of the anti-TB drugs such as pyrazinamide (PZA), the treatment is usually initiated without knowledge of the drug's susceptibility of the bacilli in many developing countries including Cambodia. $^{7-10}$

Currently, first-line drugs (FLD), including isoniazid (INH), rifampin (RIF), ethambutol (EMB) and PZA, form the core of treatment regimen for drug-susceptible $\mathrm{TB}$, while fluoroquinolones (FQ) and second-line injectable agents (SLID) are the two main classes of drugs used in the MDR-TB treatment regimen. ${ }^{11}$ Prior to 2017 , streptomycin (STM) was also included in the first-line TB treatment for retreated patients (WHO category II regimen) in many countries where conventional or molecular DST was not routinely available. ${ }^{12,13}$ However, due to its resistance and toxicity, STM is now a reserved second-line injectable agent and used as a substitute for amikacin in the MDR-TB regimen. Some FLD such as INH, EMB and PZA also play an important role in the chemotherapy of drug-resistant TB. Compared to drug-susceptible TB, treatment of MDR-TB and XDR-TB requires prolonged expensive chemotherapy, often associated with important side effects and a low success rate, $56 \%$ for MDR-TB and $39 \%$ for XDR-TB. More extreme drug resistance patterns to all available FLD and SLD drugs, ie, totally drugresistant TB (TDR), ${ }^{3}$ also known as extremely drugresistant (XXDR) by Migliori et al, ${ }^{12}$ have already been reported and were untreatable with currently available drugs. For this reason, it is of critical importance to understand how drug resistance arises, evolves and how it may be prevented. The aim of this study is to acquire phenotypic and genetic knowledge specifically on secondline anti-TB drug (SLD) resistance in Cambodia. This study was focused on M.tb clinical isolates phenotypically resistant to SLD, including pre-XDR and XDR.

\section{Materials and Methods M.tb Strain Collection and NTP Routine for MDR-TB Screening}

This retrospective cross-sectional study included FLDresistant M.tb isolates taken from the collection of clinical isolates obtained via a platform established for routine screening of MDR-TB among high-risk groups by the National Tuberculosis Program (NTP). ${ }^{13}$ Since 2012, people at risk of developing MDR-TB were screened using Xpert MTB/RIF, followed by culture and phenotypic drug susceptibility testing (pDST) for FLD. All confirmed RIF resistant (RR) or MDR-TB cases received a 24-month standardized regimen in two phases: an 8-month intensive phase and a 16-month continuation phase. The treatment contained PZA and at least 4 effective SLD including one SLID, one FQ and two oral bacteriostatic drugs selected among Ethionamide (Eto), Cycloserine (Cs) or P-aminosalicylic acid (PAS). ${ }^{14}$ The Cambodian NTP adopted the WHO-recommended MDR-TB shorter regimen in 2017 and the rapid molecular DST (GenoType MTBDRsl, Hain LifeScience, Germany) was systematically used to screen for resistance to FQ and SLID for all eligible patients for the shorter regimen. ${ }^{15}$

The National TB Reference Laboratory (NRL) of the National Centre for Tuberculosis and Leprosy Control (CENAT) and the Mycobacteriology Laboratory of the Institut Pasteur in Cambodia (IPC) are the two main DST laboratories in Cambodia. During their routine screening activities to detect drug-resistant TB among MDR-TB highrisk groups, M.tb were isolated, identified, and cryopreserved in the strain bank at the IPC and NRL. All the strains were thus tested for their susceptibility to first-line anti-TB drugs (FLD) including rifampicin (RIF; $1.0 \mu \mathrm{g} / \mathrm{mL}$ ), isoniazid (INH; $0.1 \mu \mathrm{g} / \mathrm{mL}$ ), ethambutol (EMB; $5.0 \mu \mathrm{g} / \mathrm{mL}$ ), streptomycin (STM; $1.0 \mu \mathrm{g} / \mathrm{mL}$ ) by pDST using the automated BACTEC MGIT 960 (MGIT AST SIRE kit, Becton Dickinson (BD), Sparks, MD, USA) ${ }^{16}$ STM is considered as one of the FLD since it was used in first-line TB treatment for retreated patients until the end of 2017. ${ }^{9}$ The DST results were not available for PZA. 
Patient data including age, gender, geographic area and year of registration, clinical information, FLD susceptibility profile and treatment outcomes were collected from the patients' laboratory and available medical records.

\section{DNA Preparation}

The original stocks of clinical M.tb isolates stored at $-80^{\circ}$ $\mathrm{C}$ were re-cultured on Löwenstein-Jensen (LJ) medium slants. A loopful of M.tb colonies recovered from LJ medium slants were suspended in $300 \mathrm{~mL}$ of TE buffer (10 mM Tris, $1 \mathrm{mM}$ EDTA pH 8.5) and boiled for $30 \mathrm{~min}$. The supernatant containing bacterial DNA was used as PCR template.

\section{Phenotypic Drug Susceptibility Testing (PDST)}

The pDST of M.tb to second-line anti-TB drugs was carried out in a Biosafety Level 3 laboratory at the IPC using the BACTEC'M MGIT'T 960 liquid culture system (BD, Sparks, MD, USA). The MGIT DST method was used according to the standard critical antibiotic concentrations recommended by the WHO: ofloxacin (Ofx; 2.0 $\mu \mathrm{g} / \mathrm{mL}$ ); amikacin (Am; $1.0 \mu \mathrm{g} / \mathrm{mL}$ ); kanamycin (Km; 2.5 $\mu \mathrm{g} / \mathrm{mL})$ and capreomycin $(\mathrm{Cm} ; 2.5 \mu \mathrm{g} / \mathrm{mL}) .{ }^{17}$ Based on the FLD and SLD phenotypic DST profiles, the M.tb isolates under this study were categorized into MDR, non-MDR, fluoroquinolone-resistance (FQ-R), second-line injectable drug (SLID) resistance, pre-XDR and XDR (see Box 1).

\section{Sequencing}

The main genes associated with resistance to FLD and SLD were amplified by PCR using specific primers reported in Table S1 and sequenced (Macrogen Inc., Republic of Korea and Eurofins Genomics Germany GmbH, Germany). For FLD resistance, the following genes and gene fragments were studied: the $k a t \mathrm{G}$ gene, the $i n h \mathrm{~A}$ gene coding and the inh $\mathrm{A}$ promoter (INH resistance); the rpoB gene (RIF resistance); the $r p s \mathrm{~L}$ gene and $r r s-\mathrm{F} 1$ fragment targeting $r r s 530$ and 915 loops (STM resistance); the embB gene (EMB resistance); the pncA gene and its promoter (PZA resistance). For SLD resistance (including FQ and SLID), the following genes or gene fragments were analyzed: the gyrA and gyrB genes (FQ resistance); the rrs-F2 fragment targeting the rrs 1400-1500 region (SLID resistance). ${ }^{18}$ The sequences were aligned with the M.tb H37Rv reference (GenBank NC000962.3) using the Bioedit software v7.2.6. ${ }^{19}$ To describe the mutations associated with
Box I Definition of Drug-Resistant Categories Based on Phenotypic DST Profiles

First-line drug resistance (FLD-R)*: Resistance to any first-line drugs (FLD) tested including INH, RIF, EMB and STM.

Second-line drug resistance (SLD-R): Resistance to any second-line drugs tested including fluoroquinolones (Ofx) and second-line injectable drugs ( $\mathrm{Am}, \mathrm{Km}$ and $\mathrm{Cm}$ ).

Multidrug-resistance (MDR): Resistance to at least two of the most effective anti-TB drugs, INH and RIF.

Non-MDR: Resistance to one or more FLD but not to INH and RIF at the same time.

Quadruple drug resistance (QDR): Resistance to all four first line drugs tested including INH, RIF, EMB and STM.

Fluoroquinolone resistance (FQ-R): Phenotypic resistance to any fluoroquinolone (ie Ofx in this study).

Second-line injectable drug (SLID) resistance: Phenotypic resistance to at least one of three SLID including Am, $\mathrm{Km}$ and $\mathrm{Cm}$.

Extensively drug-resistant (XDR): MDR isolates simultaneously resistant to any fluoroquinolone (ie Ofx in this study) and at least to one SLID (Am, $\mathrm{Km}$ and $\mathrm{Cm}$ ).

Pre-extensively drug-resistant (pre-XDR): MDR isolates resistant either to any FQ (ie Ofx in this study) or one SLID (Am, Km and Cm).

Note: *Phenotypic DST is not available for pyrazinamide.

Abbreviations: Am, amikacin; $\mathrm{Cm}$, capreomycin; DST, Drug susceptibility testing; $\mathrm{EMB}$, ethambutol; INH, isoniazid; $\mathrm{Km}$, kanamycin; Ofx, ofloxacin; R, resistance; RIF, rifampicin; STM, streptomycin.

resistance in the rpoB and $g y r \mathrm{~B}$ genes, a numbering system based on the Escherichia coli sequence annotation and 2002 M.tb H37Rv sequence re-annotation was used..$^{20,21}$ The interpretation of mutations related to resistance was based on resistance mutation sites reported in previous studies. ${ }^{18,22-24}$ When the results between DNA sequencing and pDST were not consistent, both methods were repeated. If the repeated result conflicted with the original data, a third round of testing was accepted as the final result.

\section{Spoligotyping}

Spoligotyping was carried out following previously described standard techniques. ${ }^{25,26}$ The spoligotype international type (SIT) was determined using the 6th version of the international genotyping database SITVIT2. ${ }^{27}$ The isolates with an undefined pattern declared as "orphans or New" were further compared with the SPOTCLUST database. ${ }^{28}$ The family assignation was retained when the probability was $\geq 95 \%$.

\section{MIRU-VNTR}

Standardized 24-locus mycobacterial interspersed repetitive unit-variable number tandem repeat (MIRU-VNTR) typing was performed by following the recommendations 
of Supply et al. ${ }^{29}$ The allelic profiles were analyzed using the MIRU-VNTRplus database (available at www.miruvntrplus.org/).

The resolution power of 24 MIRU-VNTR was determined based on the Hunter-Gaston- Discriminatory Index (HGDI). ${ }^{30}$ The method was classified as "highly discriminant" (HGDI ranged between 0.90 and 0.99), "moderately discriminant" (HGDI ranged between 0.6 and 0.9) and "poorly discriminant" (HGDI <0.6). ${ }^{31}$ The genetic diversity within sample $\left(\mathrm{H}_{\mathrm{s}}\right)$ was calculated using FSTAT version 2.9.3.2. ${ }^{32}$

\section{Phylogenetic Reconstruction}

Phylogenetic analyses were performed combining spoligotype and 24 loci MIRU-VNTR profiles and a NeighborJoining (NJ) tree was built using the Chord distance (Cavalli-Sforza and Edwards 1967) setting in the MIRUVNTRplus web-based tool to calculate the distance matrix. ${ }^{33,34}$ We included one $M$. canettii reference database isolate as outgroup. A cluster was defined as a group of two or more isolates sharing the same combined spoligotype and 24 MIRU-VNTR patterns.

\section{Results}

\section{Mycobacterium tuberculosis Population Under Study}

Among a total of 710 M.tb isolates (1 isolate per patient) collected from 2012 to 2017 and stored at the IPC and NRL banks, 309 were phenotypically resistant to at least one FLD and 401 were pan-susceptible (Figure 1). Among the 309 drug-resistant M.tb isolates, 278 were successfully subcultured on Lowenstein-Jensen (LJ) medium slants and underwent sequencing of the $g y r \mathrm{~A} / \mathrm{B}$ and/or $r r s-\mathrm{F} 2$ genes involving resistance to $\mathrm{FQ}$ and SLID, respectively. However, the sequencing results were obtained for only 272 isolates including 118 (43.4\%) MDR isolates and 154 (56.6\%) non-MDR isolates (See Table S2). Among these, 37 isolates carried mutations in the $g y r \mathrm{~A} / \mathrm{B}$ and $r r s-\mathrm{F} 2$ genes. The data on these 37 isolates are detailed in Table 1. Out of these 37 isolates, the phenotypic resistance to any SLD including Ofx, $\mathrm{Am}, \mathrm{Km}$ and $\mathrm{Cm}$ was found only for 25 isolates which were then used for genotypic study.

\section{Characteristics of Patients}

Patient data including age, gender, geographic area, year of registration and clinical information linked to the 272 isolates included in the study were collected (See Table S3).
Absence of recorded data was considered as missing data and not included in the analysis. From the available data, most of the study subjects were males $(164 / 258 ; 63.6 \%)$. The mean age at the time of diagnosis was 44.9 (95\% CI, 42.9 to 46.8 ), ranging from 15 to 83 years. The majority of patients had pulmonary TB $(215 / 220 ; 97.7 \%)$. Five patients had extrapulmonary TB including two lymph nodes TB, two TB meningitis and one pleural TB. Among the 203 pulmonary TB patients, $68.9 \%$ (140/203) were smear-positive. Most study subjects were TB cases in re-treatment (140/254; 94.5\%). The HIV status was known for only 18 patients and eight were HIV-positive patients (8/18; 44.5\%). SLD resistance TB cases were only observed among pulmonary TB patient (100\%) and a higher number of cases was observed among the 15 - to 34 -year $(32.0 \%$; $95 \%$ CI, 13.2 to 50.8$)$ and 35 - to 54 -year $(60.0 \% ; 95 \% \mathrm{CI}, 40.3$ to 79.7$)$ age groups compared to $\geq 55$-year age group $(8.0 \% ; 95 \% \mathrm{CI}$, -2.9 to 18.9 ) (See Table S3).

\section{Resistance to First-Line Drugs}

Phenotypic Resistance to FLD: INH, RIF, STM, EMB

The phenotypic resistance to FLD was available for INH, RIF, STM and EMB (See Table 1 and S2). For susceptibility to PZA, only molecular data were acquired in the framework of this study and are detailed in the following paragraph. Among the 37 isolates, 24 (64.9\%) were MDR and $13(35.1 \%)$ were non-MDR. The phenotypic resistance rate of all the clinical isolates to each FLD was as follows: INH, $83.8 \%(\mathrm{n}=31)$; RIF, 70.3\% $(\mathrm{n}=26)$; STM, $64.9 \%(\mathrm{n}=24)$ and EMB, 27.0\% $(\mathrm{n}=10)$. Among the 24 MDR isolates, $21.6 \%(\mathrm{n}=8)$ were resistant to all four FLD, defined here as "Quadruple drug-resistant" (QDR) isolates.

Mutations in the katG, inhA, rpoB, rrs-FI, rpsL and embB Genes

The mutations detected in genes involving resistance to FLD are reported in Tables 1 and 2. Among the $31 \mathrm{INH}-$ resistant isolates, $24(77.4 \%)$ harbored mutations in the kat $\mathrm{G}$ gene, $5(16.1 \%)$ in the $i n h \mathrm{~A}$ core gene and/or its promotor, and $1(3.2 \%)$ in both genes. One (3.2\%) INHresistant isolate had no mutation in either $k a t \mathrm{G}$ or inh $\mathrm{A}$. The most common mutations were kat $\mathrm{G} \mathrm{S} 315 \mathrm{~T}$ (20/30, 66.7\%), followed by inhA c-15t+S94A (3/30, $10 \%)$ and $\operatorname{inh} \mathrm{A} \mathrm{c}-15 \mathrm{t}(2 / 30,6.7 \%)$. Other mutation points were found: $k a t \mathrm{G} 3315 \mathrm{~N}$; kat $\mathrm{G}$ W149R; katG $\mathrm{W} 477 \mathrm{~L}+\mathrm{A} 479 \mathrm{P}$ and $k a t G \mathrm{~F} 720 \mathrm{~S}+i n h A \mathrm{c}-15 \mathrm{t}$ in one isolate each. For one (3.2\%) isolate, $k a t \mathrm{G}$ could not be amplified after repetitions using two pairs of primers 


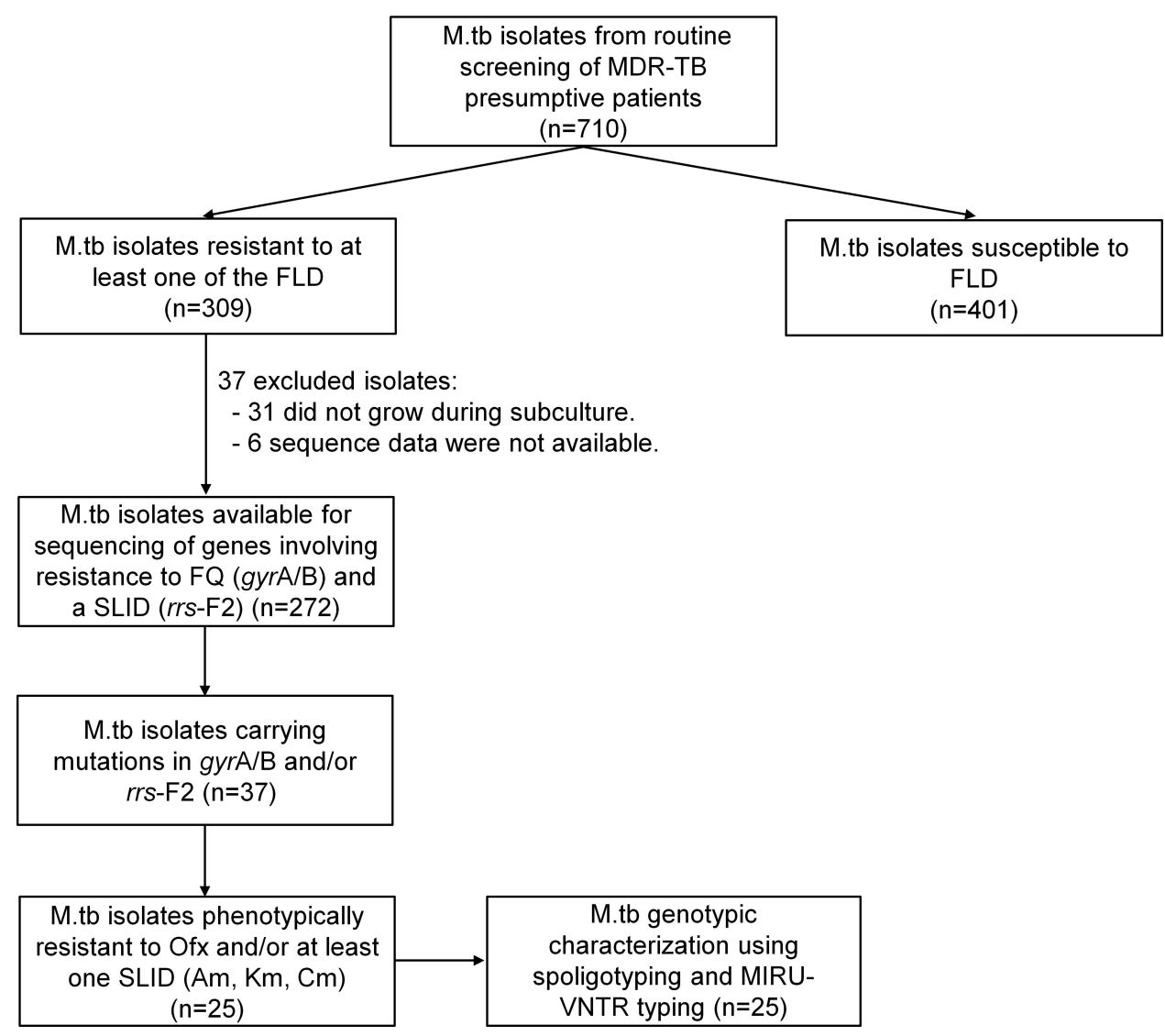

Figure I Mycobacterium tuberculosis isolate collection and analytic methods.

Abbreviations: Am, amikacin; Cm, capreomycin; FLD, first-line drugs; FQ, fluoroquinolones; Km, kanamycin; M.tb, Mycobacterium tuberculosis; MDR, multidrug-resistant; Ofx, ofloxacin; SLID, second-line injectable drugs; TB, tuberculosis.

targeting two different regions on the gene. The result suggests that the partial or completed kat $\mathrm{G}$ gene was truncated, as reported by Ramaswamy et al. ${ }^{35}$ One out of 6 INH-susceptible isolates had mutation G560S in the kat $\mathrm{G}$ gene.

Among the 26 RIF-resistant isolates, 25 (96.2\%) had mutations at least at one codon in the 81-bp $r p o \mathrm{~B}$ rifampicin resistance-determining region (RRDR) and one isolate had no mutation in the $r p o \mathrm{~B}$ gene. The most prevalent drug-resistant mutations were S531L, $\mathrm{H} 526 \mathrm{D} / \mathrm{R} / \mathrm{Y}$ and $\mathrm{D} 516 \mathrm{~V}$, which accounted for $50.0 \%$, $19.2 \%$ and $3.8 \%$, respectively. Five isolates carried double mutations; three (11.5\%) carried L511P + H526Q and two (7.7\%) were found with L511R + D516Y mutations. One isolate carried rpoB Q513-F514ins. Among the 11 RIFsusceptible isolates, one harbored mutation I572F, which was reported to appear among the mutations conferring low-level RIF resistance. ${ }^{36,37}$

All the 24 isolates resistant to STM exhibited mutations in $r p s \mathrm{~L}$ or $r r s-\mathrm{F} 1$. The mutation in $r p s L \mathrm{~K} 43 \mathrm{R}$ was the most prevalent and accounted for $50 \%$, followed by the mutations rpsL K88R and rrs-F1 a514c accounting for $29.2 \%$ and $12.5 \%$, respectively. Two other mutations, $r p s \mathrm{~L}$ K88T and rrs-F1 c905a, were found in one STMresistant isolate each.

Resistance to EMB was observed in ten isolates, among them, eight $(80 \%)$ isolates harbored mutations in the $e m b \mathrm{~B}$ gene. The most common drug-resistant mutations were observed in the embB codon M306L/V/I and $e m b \mathrm{~B}$ codon $\mathrm{F} 330 \mathrm{~S} / \mathrm{V}$, found in 5 and 2 of the 10 resistant isolates, respectively. However, four single mutations at the $e m b \mathrm{~B}$ codon Q497L/R; D354A and G406A were detected in five of the 27 EMB-susceptible isolates. Overall, 7 of the 37 isolates had contradictory results between EMB resistance phenotype and embB gene sequencing (see Table 1).

Overall, among the 37 isolates, one INH-resistant, one RIF-resistant and two EMB-resistant isolates had no mutation in the studied genes. In contrast, mutations in the katG (G560S), rpoB (I572F) and embB (D354A, G406A, Q497L and Q497R) genes were found in one 


\begin{tabular}{|c|c|c|c|c|c|c|c|c|c|c|c|c|c|c|c|}
\hline & 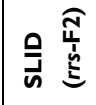 & 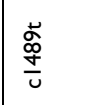 & $\xi$ & $\xi$ & $\xi$ & $\xi$ & $\xi$ & $\frac{\frac{00}{\sigma}}{\frac{\sigma}{\sigma}}$ & 5 & $\xi$ & $\xi$ & 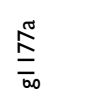 & $\xi$ & 与 & $\xi$ \\
\hline \multirow{6}{*}{ 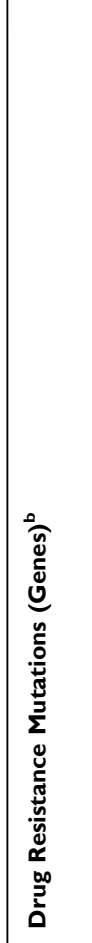 } & 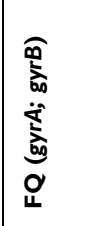 & 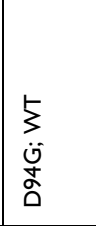 & $\begin{array}{l}5 \\
3 \\
\dot{3} \\
\dot{\alpha}\end{array}$ & 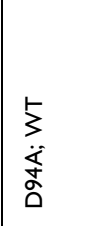 & 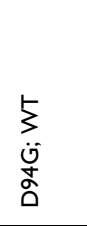 & \begin{tabular}{l}
5 \\
\multirow{5}{*}{} \\
$\infty$ \\
$\vdots$ \\
$\vdots$ \\
$\alpha$ \\
$\alpha$
\end{tabular} & 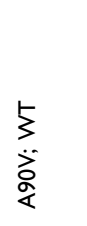 & $\begin{array}{l}\xi \\
\xi \\
\xi\end{array}$ & 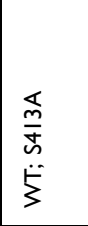 & 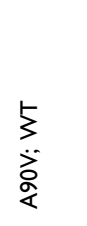 & 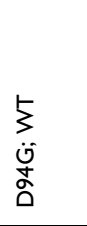 & $\begin{array}{l}\xi \\
\xi\end{array}$ & $\begin{array}{l}\xi \\
\frac{5}{\delta} \\
\frac{\dot{\alpha}}{\sigma}\end{array}$ & 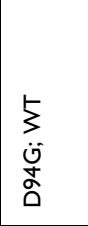 & $\begin{array}{l}5 \\
\frac{5}{10} \\
\frac{1}{0} \\
0 \\
\frac{1}{2} \\
0\end{array}$ \\
\hline & $\begin{array}{l}\widehat{\widehat{V}} \\
\text { 产 } \\
\text { N }\end{array}$ & 㐔 & $\frac{\vec{\omega}}{\frac{\vec{T}}{\alpha}}$ & $\xi$ & $\stackrel{\circ}{\Xi}$ & $\frac{\frac{\omega}{\underline{T}}}{\frac{\pi}{\sigma}}$ & $\frac{\frac{\omega}{\sigma}}{\frac{\pi}{\sigma}}$ & 5 & 尊 & $\underset{\frac{1}{4}}{\gtrless}$ & $\xi$ & $\xi$ & $\underset{\substack{x \\
x}}{\vec{\lambda}}$ & 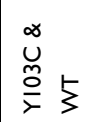 & $\frac{\frac{0}{m}}{\frac{m}{>}}$ \\
\hline & 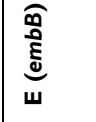 & 商 & $\xi$ & 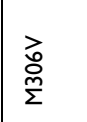 & 首 & $\xi$ & 㶾 & $\xi$ & 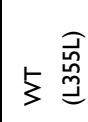 & $\begin{array}{l}\overline{\mathrm{o}} \\
\overline{\tilde{o}}\end{array}$ & $\xi$ & $\xi$ & $\begin{array}{l}\frac{\alpha}{0} \\
\text { ơ } \\
\text { d }\end{array}$ & $\xi$ & 总 \\
\hline & 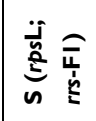 & 㸺寺 & 蔓走 & 羊与 & 永寺 & 蒿 & 兽卢 & 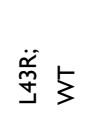 & 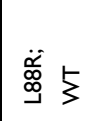 & 芳章 & 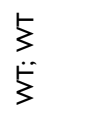 & 芌苔 & 兽 & 芦喜 & 晜寺 \\
\hline & 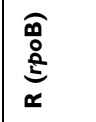 & 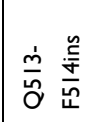 & 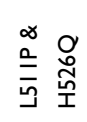 & 离 & 离 & 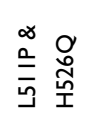 & 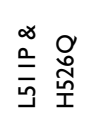 & 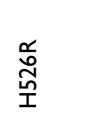 & $\xi$ & 兽 & 兽 & $\xi$ & 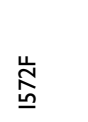 & 窇 & 莺 \\
\hline & 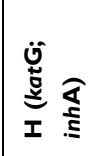 & $\begin{array}{l}\frac{5}{3} \\
\stackrel{-1}{5} \\
\frac{5}{n}\end{array}$ & 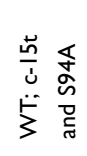 & $\begin{array}{l}3 \\
\stackrel{5}{3} \\
\frac{-5}{\omega} \\
\tilde{\omega}\end{array}$ & 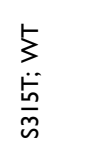 & 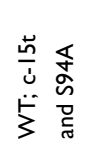 & 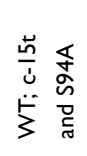 & 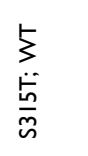 & $\begin{array}{l}5 \\
\stackrel{5}{0} \\
\text { 兽 }\end{array}$ & 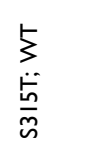 & $\begin{array}{l}\xi \\
\xi\end{array}$ & 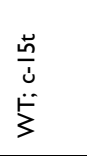 & 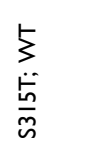 & 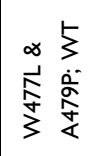 & 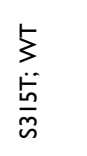 \\
\hline \multirow{2}{*}{ 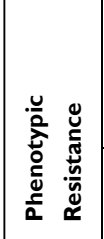 } & $\overrightarrow{\vec{v}}$ & $\begin{array}{l}\dot{E} \\
\dot{z} \\
\frac{\dot{x}}{\partial} \underline{E}\end{array}$ & 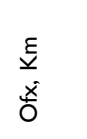 & 希 & x & xo & ox & $\begin{array}{l}\underline{E} \\
\frac{E}{\xi}\end{array}$ & 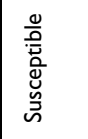 & xx & ox & 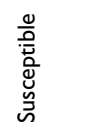 & ox & x & ỡ \\
\hline & 竎 & 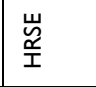 & $\stackrel{\mathscr{x}}{\underline{I}}$ & \begin{tabular}{|l} 
岀 \\
蒫
\end{tabular} & 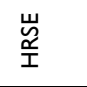 & $\stackrel{\mathscr{Q}}{\underline{I}}$ & 亗 & $\stackrel{\mathscr{\underline { I }}}{\underline{\underline{I}}}$ & $\infty$ & 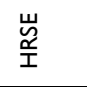 & $\propto$ & $\underline{\underline{x}}$ & 모 & 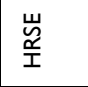 & $\stackrel{\mathscr{O}}{\underline{x}}$ \\
\hline 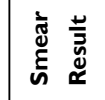 & & ơ & ס̊ & ơ & ○ & $\frac{s}{z}$ & 荘 & ŏ & 苂 & סू & 。ั & $\frac{\pi}{z}$ & ŏ & ŏ & 음 \\
\hline \multicolumn{2}{|c|}{ 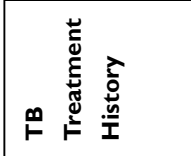 } & 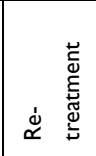 & 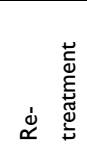 & 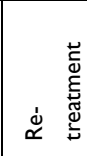 & 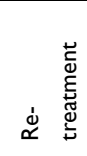 & 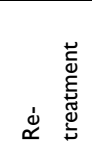 & 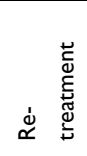 & $\stackrel{\infty}{\stackrel{\infty}{I}}$ & 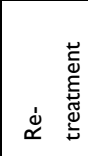 & 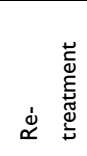 & 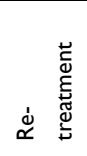 & 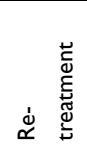 & 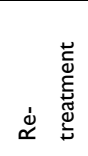 & 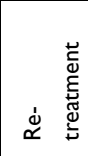 & 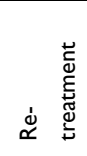 \\
\hline \multicolumn{2}{|l|}{ 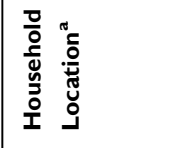 } & $\begin{array}{l}\text { 조 } \\
\text { 咅 }\end{array}$ & $\frac{\underline{T}}{\frac{1}{\dot{y}}}$ & \begin{tabular}{|l} 
I \\
至
\end{tabular} & 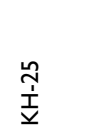 & $\begin{array}{l}\text { 艿 } \\
\text { 王 }\end{array}$ & $\frac{\frac{\pi}{T}}{\frac{\dot{I}}{\underline{y}}}$ & $\begin{array}{l}\hat{O} \\
\text { 王 }\end{array}$ & \begin{tabular}{|l}
$\hat{O}$ \\
咅
\end{tabular} & 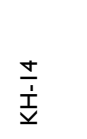 & $\frac{T}{\frac{T}{\dot{I}}}$ & 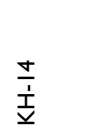 & $\frac{\stackrel{N}{I}}{\frac{\dot{I}}{\underline{y}}}$ & 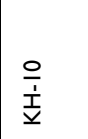 & 喜 \\
\hline \multicolumn{2}{|l|}{ 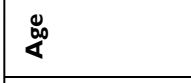 } & $\infty$ & $\approx$ & $\bar{n}$ & in & 6 & $\stackrel{\circ}{m}$ & q & 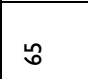 & $\stackrel{\sim}{\sim}$ & $\bar{\sigma}$ & $R$ & $\tilde{m}$ & in & $\tilde{n}$ \\
\hline \multicolumn{2}{|l|}{ 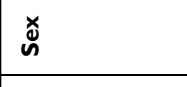 } & $\Sigma$ & 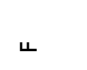 & ㄴ & $\Sigma$ & $\Sigma$ & 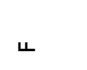 & $\Sigma$ & $\Sigma$ & $\Sigma$ & $\Sigma$ & $\Sigma$ & ${ }_{4}$ & $\Sigma$ & $\Sigma$ \\
\hline \multicolumn{2}{|l|}{ 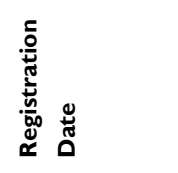 } & 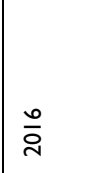 & $\stackrel{\sim}{\circ}$ & 商 & iे & $\overline{\frac{N}{2}}$ & $\stackrel{n}{\grave{N}}$ & $\frac{n}{i}$ & $\stackrel{n}{\grave{a}}$ & $\stackrel{\underline{n}}{\bar{N}}$ & $\stackrel{n}{\bar{N}}$ & $\stackrel{ \pm}{\stackrel{ \pm}{*}}$ & $\bar{\lambda}$ & $\stackrel{+}{\stackrel{亠}{a}}$ & $\bar{i}$ \\
\hline \multicolumn{2}{|l|}{ 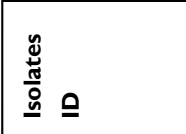 } & $\begin{array}{l}\bar{s} \\
\alpha_{1}^{\prime}\end{array}$ & $\begin{array}{l}\tilde{z} \\
\sigma_{1}^{\prime}\end{array}$ & 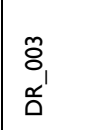 & 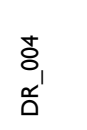 & $\begin{array}{l}\hat{o} \\
o \\
o \\
o\end{array}$ & $\begin{array}{l}0 \\
\tilde{o}_{1} \\
u_{0}^{\prime}\end{array}$ & 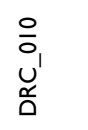 & 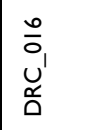 & $\begin{array}{l}\overline{\sigma_{1}} \\
\text { ö } \\
\tilde{u}^{\prime}\end{array}$ & 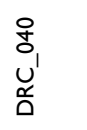 & $\begin{array}{l}\text { o̊ } \\
u_{1} \\
\stackrel{0}{0}\end{array}$ & $\begin{array}{l}\tilde{o} \\
\mathrm{o}^{\prime} \\
\bigcup_{0}^{\prime}\end{array}$ & 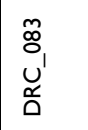 & 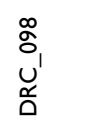 \\
\hline
\end{tabular}




\begin{tabular}{|c|c|c|c|c|c|c|c|c|c|c|c|c|c|c|}
\hline$\xi$ & $\xi$ & $\xi$ & $\xi$ & $\xi$ & $\xi$ & $\xi$ & $\xi$ & $\xi$ & $\xi$ & $\xi$ & $\frac{\frac{0}{\sigma}}{\frac{\sigma}{\sigma}}$ & $\xi$ & $\xi$ & $\xi$ \\
\hline 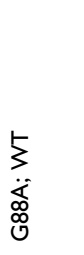 & $\begin{array}{l}5 \\
\text { ơ } \\
\text { ơ } \\
\text { an }\end{array}$ & $\begin{array}{l}5 \\
\vdots \\
\text { ơ } \\
\text { ô }\end{array}$ & $\begin{array}{l}\overrightarrow{\overrightarrow{0}} \\
\underline{\underline{b}} \\
\ddot{\xi}\end{array}$ & 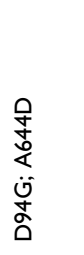 & 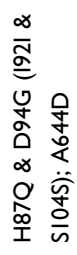 & 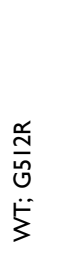 & 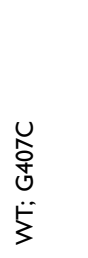 & 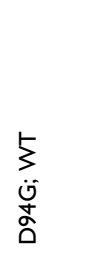 & $\begin{array}{l}5 \\
\text { ơ } \\
\text { ờ }\end{array}$ & 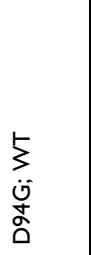 & $\begin{array}{l}5 \\
\check{\xi}\end{array}$ & $\begin{array}{l}5 \\
\text { ơ } \\
\text { ờ } \\
\text { on }\end{array}$ & 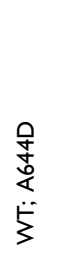 & 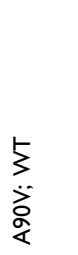 \\
\hline$\xi$ & 窇 & 5 & $\xi$ & $\frac{\infty 00}{\bar{d}}$ & $\xi$ & $\xi$ & $\frac{\text { no }}{\bar{d}}$ & 5 & 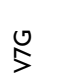 & $\stackrel{\stackrel{N}{~}}{\lessgtr}$ & $\xi$ & $\stackrel{u}{\not 凶}$ & 5 & $\xi$ \\
\hline$\xi \stackrel{\text { 今. }}{\stackrel{\widehat{H}}{2}}$ & $\xi$ & $\xi$ & 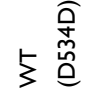 & $\xi$ & $\xi$ & $\xi$ & 蚛 & $\xi$ & 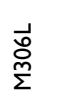 & $\begin{array}{l}\overrightarrow{\mathrm{o}} \\
\stackrel{m}{\Sigma}\end{array}$ & $\xi$ & $\begin{array}{l}\vec{े} \\
\text { m }\end{array}$ & $\xi$ & $\xi$ \\
\hline $\begin{array}{l}5 \\
\stackrel{5}{3}\end{array}$ & 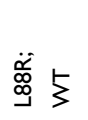 & 芦 & $\begin{array}{l}5 \\
\stackrel{5}{3}\end{array}$ & 苂々 & 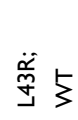 & $\begin{array}{l}5 \\
\vdots \\
3\end{array}$ & $\begin{array}{l}5 \\
\vdots \\
\xi\end{array}$ & $\stackrel{\ddot{\tilde{g}}}{\lessgtr} \lessgtr$ & $\begin{array}{l}5 \\
\vdots \\
\xi\end{array}$ & $\begin{array}{l}5 \\
\dot{5} \\
3\end{array}$ & $\begin{array}{l}5 \\
\vdots \\
\xi\end{array}$ & 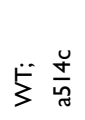 & $\stackrel{\ddot{\tilde{v}}}{\lessgtr} \lessgtr$ & $\begin{array}{l}5 \\
\vdots \\
3\end{array}$ \\
\hline $\overrightarrow{\vec{n}}$ & 馬 & $\xi$ & $\overrightarrow{\vec{n}}$ & $\overrightarrow{\vec{N}}$ & $\frac{\partial}{\mathrm{n}}$ & $\xi$ & 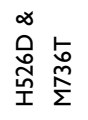 & 5 & 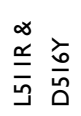 & 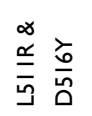 & $\overrightarrow{\underline{N}}$ & 总 & 5 & $\begin{array}{l}\text { Q } \\
\text { 现 }\end{array}$ \\
\hline $\begin{array}{l}5 \\
\xi\end{array}$ & $\begin{array}{l}\sum \\
\frac{5}{n} \\
\frac{\dot{n}}{n}\end{array}$ & $\begin{array}{l}5 \\
\breve{5}\end{array}$ & $\begin{array}{l}5 \\
\ddot{\xi}\end{array}$ & $\begin{array}{l}\frac{5}{\dot{5}} \\
\frac{\dot{n}}{\tilde{m}}\end{array}$ & $\begin{array}{l}5 \\
\stackrel{5}{\cdot n} \\
\frac{\tilde{n}}{n}\end{array}$ & 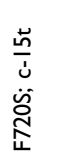 & $\begin{array}{l}\sum \\
\ddot{n} \\
\frac{\underline{m}}{m}\end{array}$ & $\begin{array}{l}5 \\
\xi \\
\xi\end{array}$ & $\begin{array}{l}\vdots \\
\frac{\mathfrak{5}}{m} \\
\dot{n}\end{array}$ & $\begin{array}{l}\vdots \\
\stackrel{5}{n} \\
\frac{\mathscr{n}}{n}\end{array}$ & $\begin{array}{l}\vdots \\
\stackrel{5}{\underline{n}} \\
\frac{\ddot{n}}{n}\end{array}$ & $\begin{array}{l}5 \\
3 \\
\frac{5}{5} \\
\frac{5}{n}\end{array}$ & $\begin{array}{l}5 \\
3 \\
\frac{5}{n} \\
\frac{5}{n}\end{array}$ & 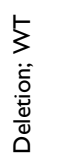 \\
\hline ỡ & ํㅗㅇ & ํㅡㅇ & 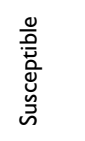 & ํㅡㅇ & 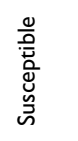 & 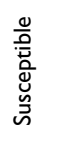 & 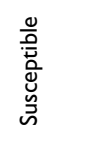 & ํㅡㅇ & ํㅡㅇ & ํㅗㅇ & $\begin{array}{l}\dot{E} \\
\underline{\underline{\xi}} \\
\dot{\xi}\end{array}$ & ํㅡㅇ & 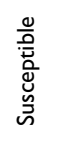 & ญั㐅 \\
\hline$\propto$ & 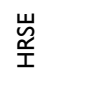 & $n$ & $\stackrel{\mathscr{x}}{\underline{I}}$ & $\stackrel{\mathscr{\mu}}{I}$ & 亗 & I & $\stackrel{\propto}{\underline{I}}$ & $n$ & 嵳 & 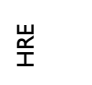 & $\stackrel{\propto}{\underline{I}}$ & 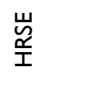 & $\underline{\underline{I}}$ & $\stackrel{\propto}{\underline{I}}$ \\
\hline ŏ & о̆ & ⿹ㅣ & o̊ & 咭 & 比 & 咭 & ơ & ơ & 比 & ○్ & ŏ & ర్ & 比 & ఝั \\
\hline \& & 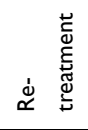 & 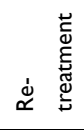 & 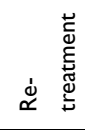 & 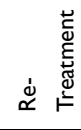 & 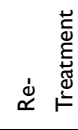 & ¿ & 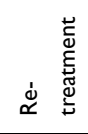 & 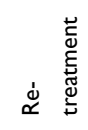 & 离 & 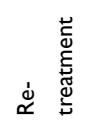 & $\underset{⿱ 亠 䒑}{\stackrel{n}{\mid}}$ & 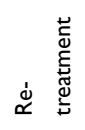 & $\mathbb{Z}$ & 它 \\
\hline 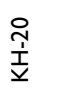 & 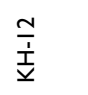 & $\begin{array}{l}\infty \\
\stackrel{\infty}{1} \\
\text { I⿱亠䒑 }\end{array}$ & $\begin{array}{l}\text { O̊ } \\
\text { 章 }\end{array}$ & 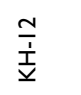 & $\begin{array}{l}\text { ò } \\
\text { 亲 }\end{array}$ & $\begin{array}{l}\bar{T} \\
\stackrel{\underline{1}}{\underline{y}}\end{array}$ & $\frac{\circ}{\frac{\mathrm{O}}{\frac{1}{\mathrm{y}}}}$ & $\frac{\frac{t}{T}}{\frac{I}{\frac{1}{2}}}$ & $\begin{array}{l}\stackrel{\infty}{0} \\
\stackrel{+}{\frac{1}{2}}\end{array}$ & $\frac{\mathfrak{T}}{\frac{1}{\dot{I}}}$ & $\begin{array}{l}\bar{T} \\
\text { 亲 }\end{array}$ & 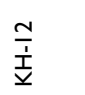 & 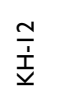 & 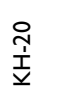 \\
\hline$\hat{N}$ & $f$ & $\approx$ & $\tilde{0}$ & $\stackrel{\circ}{\circ}$ & $\hat{\lambda}$ & $\pi$ & $\stackrel{\infty}{\sim}$ & in & $\stackrel{\infty}{q}$ & $\bar{n}$ & 이 & F & $\mathbb{z}$ & $q$ \\
\hline$\Sigma$ & $\Sigma$ & 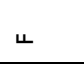 & $\longleftarrow$ & ч & $\Sigma$ & $\Sigma$ & น & $\Sigma$ & $\Sigma$ & ч & $\Sigma$ & $\Sigma$ & $\mathbb{z}$ & 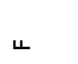 \\
\hline$\frac{\text { a }}{9}$ & $\stackrel{+}{\grave{n}}$ & $\stackrel{+}{\circ}$ & $\frac{m}{i}$ & $\frac{m}{\bar{i}}$ & $\frac{m}{a}$ & $\frac{m}{\bar{i}}$ & $\frac{m}{i}$ & $\frac{m}{a}$ & $\stackrel{\sim}{\circ}$ & $\stackrel{\circ}{\circ}$ & $\frac{\pi}{2}$ & $\frac{\pi}{2}$ & $\frac{n}{\grave{n}}$ & $\stackrel{+}{\grave{N}}$ \\
\hline $\begin{array}{l}\stackrel{o}{0} \\
U^{\prime} \\
\stackrel{o}{\Delta}\end{array}$ & $\begin{array}{l}\bar{\Xi} \\
U^{\prime} \\
\stackrel{\sim}{\Delta}\end{array}$ & 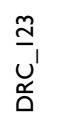 & $\begin{array}{l}\bar{m} \\
u^{\prime} \\
\stackrel{o}{\Delta}\end{array}$ & $\begin{array}{l}\hat{m} \\
u^{\prime} \\
\stackrel{o}{\Delta}\end{array}$ & 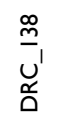 & $\begin{array}{l}\bar{\Xi} \\
U^{\prime} \\
\stackrel{\mathscr{\alpha}}{\Delta}\end{array}$ & 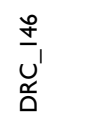 & $\begin{array}{l}\stackrel{0}{\circ} \\
u^{\prime} \\
\stackrel{\Delta}{0}\end{array}$ & $\begin{array}{l}\hat{I} \\
U^{\prime} \\
\stackrel{\Delta}{0}\end{array}$ & $\begin{array}{l}\stackrel{\varrho}{\sigma} \\
u^{\prime} \\
\stackrel{o}{\alpha}\end{array}$ & 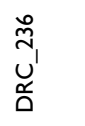 & $\begin{array}{l}\overline{y_{1}} \\
U^{\prime} \\
\stackrel{\mathscr{\Delta}}{\Delta}\end{array}$ & $\begin{array}{l}\stackrel{\infty}{\Delta_{1}} \\
\bar{\alpha} \Delta\end{array}$ & $\begin{array}{l}\stackrel{\sim}{\bar{o}} \\
\bar{\alpha} \\
\bar{\alpha}\end{array}$ \\
\hline
\end{tabular}




\begin{tabular}{|c|c|c|c|c|c|c|c|c|c|c|}
\hline \multirow{7}{*}{ 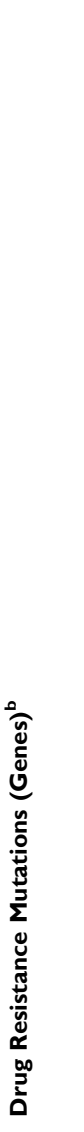 } & 号 & 5 & 5 & 5 & $\xi$ & $\xi$ & $\frac{\frac{a 0}{\alpha}}{\frac{a}{\pi}}$ & 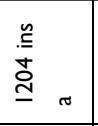 & 5 & 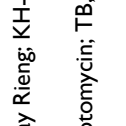 \\
\hline & 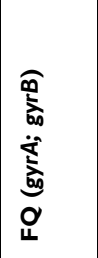 & $\begin{array}{l}5 \\
5 \\
0 \\
0 \\
0 \\
0 \\
0 \\
0 \\
0 \\
0\end{array}$ & $\begin{array}{l}5 \\
\text { i⿱ } \\
\text { o }\end{array}$ & 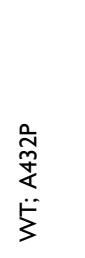 & $\begin{array}{l}5 \\
\stackrel{5}{8} \\
\ddot{8} \\
\dot{5}\end{array}$ & 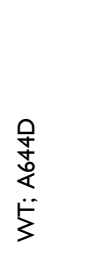 & $\begin{array}{l}5 \\
\vdots \\
\text { ờ } \\
0\end{array}$ & $\begin{array}{l}\xi \\
\xi\end{array}$ & 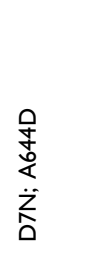 & 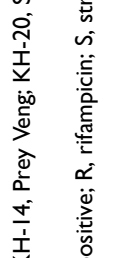 \\
\hline & $\begin{array}{l}\widehat{T} \\
\text { to } \\
\text { N }\end{array}$ & $\frac{0}{m}$ & $\xi$ & 5 & $\xi$ & $\xi$ & 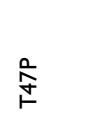 & $\xi$ & 5 & 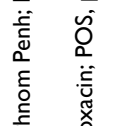 \\
\hline & 存 & 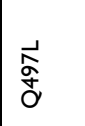 & $\xi$ & $\xi$ & $\xi$ & 5 & 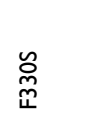 & $\xi$ & 5 & 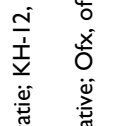 \\
\hline & 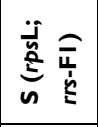 & 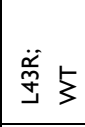 & 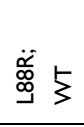 & $\begin{array}{l}5 \\
5 \\
5\end{array}$ & $\begin{array}{l}5 \\
5 \\
3\end{array}$ & $\stackrel{\ddot{\tilde{m}}}{\Xi}$ 々 & 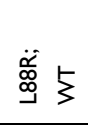 & $\begin{array}{l}5 \\
5 \\
5\end{array}$ & $\begin{array}{l}5 \\
5 \\
5\end{array}$ & 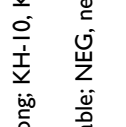 \\
\hline & 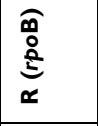 & $\overrightarrow{\bar{N}}$ & 5 & $\xi$ & 5 & 5 & 总 & 5 & 总 & 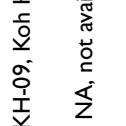 \\
\hline & 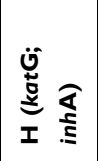 & $\begin{array}{l}5 \\
\stackrel{5}{n} \\
\frac{\omega}{n} \\
\tilde{n}\end{array}$ & $\begin{array}{l}5 \\
\xi\end{array}$ & $\begin{array}{l}5 \\
\frac{5}{n} \\
\frac{n}{n}\end{array}$ & 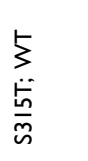 & $\begin{array}{l}5 \\
\frac{5}{n} \\
\frac{5}{n}\end{array}$ & $\begin{array}{l}5 \\
\stackrel{5}{5} \\
\frac{n}{n}\end{array}$ & $\begin{array}{l}\frac{\ddot{n}}{\dot{s}} \\
\stackrel{\vec{H}}{\xi}\end{array}$ & 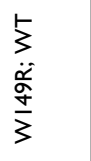 & 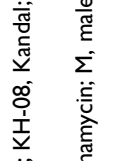 \\
\hline 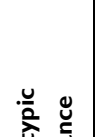 & 耍 & 希 & ํㅡㅇ & 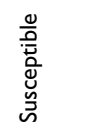 & $\begin{array}{l}\frac{0}{\overline{0}} \\
\frac{\overline{0}}{\grave{U}} \\
\bar{\omega}\end{array}$ & 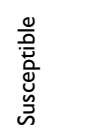 & $\begin{array}{ll}\dot{E} & E \\
\dot{X} & \tilde{x} \\
\tilde{X} & \underline{E}\end{array}$ & 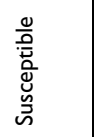 & 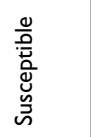 & 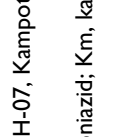 \\
\hline 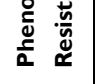 & 㟒 & $\begin{array}{l}\mathscr{\tilde { I }} \\
\stackrel{\dddot{I}}{\mathbf{n}}\end{array}$ & $n$ & $\stackrel{\propto}{\underline{I}}$ & $I$ & 또 & $\begin{array}{l}\text { Uू } \\
\stackrel{\underline{I}}{I}\end{array}$ & $I$ & $\stackrel{\mathscr{I}}{\underline{I}}$ & \\
\hline 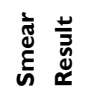 & & ơ & 适 & $\S$ & 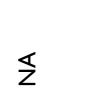 & $\S$ & ơ & $\overleftarrow{z}$ & ర్ & 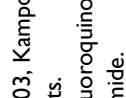 \\
\hline 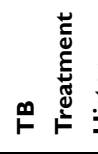 & & 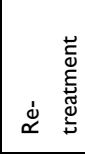 & 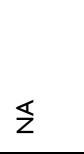 & 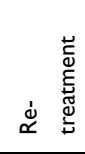 & 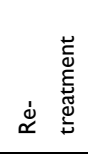 & 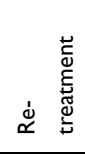 & 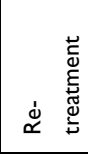 & 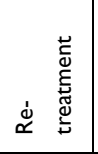 & 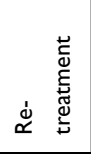 & 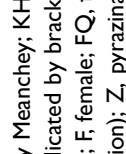 \\
\hline 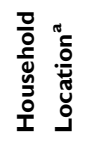 & & $\frac{\frac{T}{1}}{\frac{1}{2}}$ & 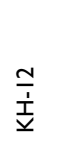 & $\begin{array}{l}\text { ồ } \\
\text { 立 }\end{array}$ & 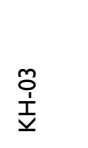 & 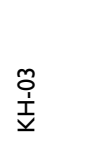 & $\frac{\stackrel{2}{1}}{\frac{1}{1}}$ & $\frac{\dddot{T}}{\frac{1}{\dot{I}}}$ & $\begin{array}{l}\text { ò } \\
\text { 京 }\end{array}$ & 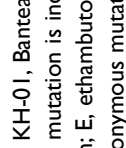 \\
\hline$\underset{8}{\stackrel{0}{4}}$ & & $\tilde{n}$ & กี & $\infty$ & 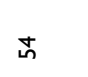 & $\dddot{\sim}$ & f & 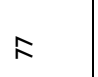 & in & \\
\hline$\stackrel{x}{\emptyset}$ & & $\Sigma$ & $\Sigma$ & ч & $\Sigma$ & ч & $\Sigma$ & $\Sigma$ & 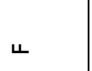 & \\
\hline 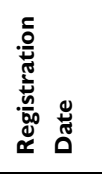 & & $\frac{4}{2}$ & $\frac{\pi}{8}$ & $\stackrel{m}{\bar{i}}$ & $\frac{m}{i}$ & ⿳亠口冋 & $\overline{\bar{i}}$ & $\stackrel{\circ}{\circ}$ & $\bar{i}$ & 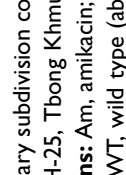 \\
\hline 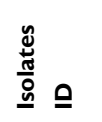 & & $\begin{array}{l}\stackrel{8}{\alpha} \\
\bar{\alpha} \\
\bar{\alpha}\end{array}$ & $\begin{array}{l}\overline{\delta_{1}} \\
\bar{\alpha}_{1}\end{array}$ & 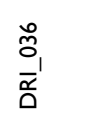 & $\begin{array}{l}\widetilde{o}_{1} \\
\overline{\tilde{\alpha}}_{\Delta}\end{array}$ & $\begin{array}{l}\stackrel{8}{1} \\
\bar{\alpha}^{\prime}\end{array}$ & $\begin{array}{l}\overline{\underline{n}} \\
\bar{\alpha}\end{array}$ & 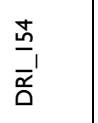 & $\begin{array}{l}\frac{\infty}{1} \\
\frac{\bar{\alpha}}{\Delta}\end{array}$ & 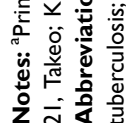 \\
\hline
\end{tabular}


Table 2 Mutation Patterns in Genes Involved in Resistance to First- and Second-Line Drugs

\begin{tabular}{|c|c|c|c|}
\hline Drug & Gene & Mutation Patterns in Drug Resistant Isolates ${ }^{a}$ & $\begin{array}{l}\text { Mutation Patterns in Drug } \\
\text { Susceptible Isolates }^{\mathbf{a}}\end{array}$ \\
\hline RIF & rpoB & $\begin{array}{l}\text { D5I6V; H526D; H526R; H526Y; S53IL; L5I IP \& } \\
\text { H526Q; H526D \& M736T; } \\
\text { L5IIR \& D5I6Y; Q5I3-F5I4ins; S53IL \& L635P }\end{array}$ & $1572 \mathrm{~F}$ \\
\hline \multirow[t]{3}{*}{ INH } & katG & $\begin{array}{l}\text { WI49R; S3I5T; S3I5N; } \\
\text { W477L \& A479P; Gene deletion }\end{array}$ & G560S \\
\hline & $\begin{array}{l}\text { inhA gene and } \\
\text { promoter region }\end{array}$ & $c-15 t ; c-15 t$ and S94A & \\
\hline & $k a t \mathrm{G}+\operatorname{inh} \mathrm{A}$ & $F 720 S+c-15 t$ & \\
\hline EMB & embB & M306I; M306L; M306V; D328Y; F330S; F330V & D354A; G406A; Q497R; Q497L \\
\hline \multirow[t]{2}{*}{ STM } & $r p s L$ & K43R; K88R; K88T & \\
\hline & $r r s-\mathrm{FI}^{\mathrm{b}}$ & a5 I4c; c905a & \\
\hline \multirow[t]{3}{*}{ Ofx } & gyrA & G88A, A90V, S9IP, D94A, D94G, & \\
\hline & gyrB & & $\begin{array}{l}\mathrm{G} 407 C^{c}, S 4 I 3 A^{c}, A 432 P^{c}, G 5 I 2 R^{c} \\
K 600 T^{c}, I 60 I L^{c}, A 644 D^{c}\end{array}$ \\
\hline & $g y r A+g y r B$ & $D 94 G+A 644 D^{a}$ & $\begin{array}{l}D 7 N a+A 644 D^{c} \\
H 87 Q \& D 94 G+A 644 D^{c}\end{array}$ \\
\hline $\begin{array}{l}\text { SLID }(\mathrm{Am}, \mathrm{Km} \\
\text { or } \mathrm{Cm})\end{array}$ & $r r s-F 2^{d}$ & $\mathrm{a}|40 \mathrm{Ig}, \mathrm{a}| 40 \mathrm{Ig}, \mathrm{cl} 489 \mathrm{t}$ & gII77a, 1204 ins a \\
\hline $\mathrm{PZA}^{\mathrm{e}}$ & $\begin{array}{l}\text { pncA gene and } \\
\text { promoter region }\end{array}$ & \multicolumn{2}{|c|}{$\begin{array}{l}\text { a-I Ig; V7G; D8E; LI 9P; L35P; T47P; H7IY; YI03C; SI04R; VI3IG; AI7IE; AI7IV; partial pncA gene } \\
\text { deletion }\end{array}$} \\
\hline
\end{tabular}

Notes: ${ }^{a}$ Drug susceptibility patterns were obtained using the BACTEC MGIT 960 method used according to the standard critical antibiotic concentrations recommended

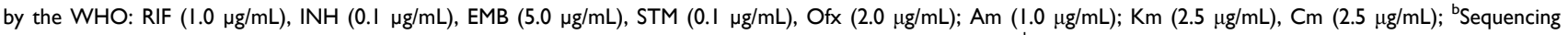
targeting Loops 530 and 915 of the rrs gene; 'Mutation outside quinolone resistance-determining regions; 'Sequencing targeting the $1400-1500$ regions of rrs gene; ésusceptibility to PZA is not available.

Abbreviations: Am, amikacin; Cm, capreomycin; EMB, ethambutol; INH, isoniazid; Km, kanamycin; Ofx, ofloxacin; PZA, pyrazinamide; RIF, rifampicin; SLID, second-line injectable agents; STM, streptomycin.

INH-susceptible, one RIF-susceptible and 5 EMBsusceptible isolates, respectively.

\section{Mutation in the pncA Gene (PZA Resistance)}

Overall, mutations in the $p n c \mathrm{~A}$ gene and its promoter were found in $18 / 37$ (48.6\%) of the isolates, including $16 / 24$ (66.7\%) MDR isolates and 2/13 (15.4\%) non-MDR isolates. Seven out of eight $(87.5 \%)$ QDR isolates had mutations in the pncA gene. Sequencing revealed high SNP (single-nucleotide polymorphism) diversity in the pnc A gene (see Table 2). In total, 13 different mutation patterns were identified, among them, 12 mutation points were found in 17 isolates and one isolate with a partial pncA gene. All the 12 mutations and the deletion had been previously documented in PZA resistant isolates. ${ }^{18,24}$ The most common mutation was $\mathrm{A} 171 \mathrm{E}$ found in three isolates
(16.7\%), followed by $\mathrm{V} 7 \mathrm{G}, \mathrm{V} 131 \mathrm{G}$ and a-11g in two isolates each $(11.1 \%)$.

\section{Resistance to Second-Line Drugs (SLD) Mutations in the gyrA and gyrB Genes}

Among the 37 isolates under study, mutations in the gyrA gene were found in 25 isolates $(67.6 \%)$ (see Tables 1 and 2). Twenty-three out of $25(92.0 \%)$ isolates had a single mutation in the quinolone resistance-determining region (QRDR) of gyrA and the most recorded mutation was D94G $(15 / 23,62.5 \%)$, followed by A90V $(5 / 23$, $21.7 \%)$, D94A (1/23, 4.3\%), S91P (1/23, 4.3\%) and G88A (1/23, 4.3\%). Among two other isolates, one harbored a double mutation in the QRDR region of gyrA (D94G and H87Q) and another a single point D7N mutation outside the QRDR region. 
Mutations in the gyr B gene were observed in 11 isolates (29.7\%) and all of them were located outside the QRDR region. The most common mutation observed in the gyrB gene was A644D (5/11, 45.5\%). Other point mutations were found: G407C, S413A, A432P, G512R, $\mathrm{K} 600 \mathrm{~T}$ and $\mathrm{I} 601 \mathrm{~L}$ in one isolate each. The $\operatorname{gyr} B \mathrm{~A} 644 \mathrm{D}$ mutation was found together with mutations in $g y r \mathrm{~A}$ in three isolates (see Table 2).

\section{Mutations in the rrs-F2 Gene}

Among the 37 isolates under study, six isolates exhibited mutations in the rrs-F2 gene (see Tables 1 and 2). Among them, mutation a1401g was found in three (50\%) isolates, and three other mutations including g1177a, 1204 ins a and c1489t, that have not been reported elsewhere, were found in one isolate each.

\section{DST for SLD on the 37 Strains with Mutations in the} gyrA/B and/or rrs-F2 Genes

In total, 37 isolates carried mutations in genes linked to SLD. The 37 isolates under study were tested for their susceptibility to second-line drugs including FQ (Ofx) and SLID (Km, Am and $\mathrm{Cm}$ ); the results are shown in Table 1. Among them, only 25 isolates including 19 MDR and 6 non-MDR exhibited resistance to at least one SLD and were used for genotypic analysis. Among non-MDR isolates, Ofx-resistance was found in all the 6 isolates including isolates with mono-resistance to STM $(n=3)$ and to RIF $(n=2)$ and poly-resistance to INH and STM ( $\mathrm{n}=1)$. Resistance to any of the SLID was not observed among non-MDR isolates. Among the 19 MDR isolates, 16 (66.7\%) were resistant to either Ofx $(14 ; 87.5 \%)$ or at least one SLID $(2 ; 12.5 \%)$ corresponding to the pre-XDR profile, and three (12.5\%) MDR isolates were resistant to Ofx and at least one SLID simultaneously corresponding to XDR isolates.

All 23 isolates with a single mutation in the $\mathrm{QRDR}$ region of the gyrA gene were resistant to Ofx. A double mutation (D94G and H87Q) and a single point D7N mutation outside the QRDR region were found in FQ-susceptible isolates. Ten out of 11 isolates with $g y r B$ mutation were susceptible to Ofx. One isolate with the gyrB A644D mutation was found in one Ofx-resistant isolate, together with mutations in gyrA D94G, known to be FQ determinant.

Three isolates with the rrs-F2 a1401g mutation were resistant to at least one SLID by pDST; two isolates were resistant to $\mathrm{Am}, \mathrm{Km}$ and $\mathrm{Cm}$ and one resistant to $\mathrm{Am}$ and $\mathrm{Km}$ (see Table 1). Among the isolates with the 3 new mutations, the isolate with the c1489t substitution was resistant to Am and $\mathrm{Km}$, while the isolates with the two
Table 3 Proportion of Resistance Phenotypes Among Beijing and Non-Beijing Genotypes

\begin{tabular}{|c|c|c|c|}
\hline $\begin{array}{l}\text { Phenotypic Drug } \\
\text { Resistance }\end{array}$ & $\begin{array}{l}\text { Beijing, } \\
\text { n (\%) }\end{array}$ & $\begin{array}{l}\text { Non- } \\
\text { Beijing, } \\
\text { n (\%) }\end{array}$ & $\begin{array}{l}\text { Total, } \\
\text { n (\%) }\end{array}$ \\
\hline XDR-TB & $3(100.0)$ & 0 & 3 \\
\hline FQ-resistant; pre-XDR-TB & II (78.6) & $3(2 I .4)$ & 14 \\
\hline $\begin{array}{l}\text { FQ-resistant; neither XDR- } \\
\text { nor pre-XDR-TB }\end{array}$ & $4(66.7)$ & $2(33.3)$ & 6 \\
\hline SLID-resistant; pre-XDR-TB & I (50.0) & I (50.0) & 2 \\
\hline Total & $19(76.0)$ & $6(24.0)$ & 25 \\
\hline
\end{tabular}

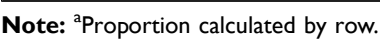

Abbreviations: $\mathrm{FQ}$, fluoroquinolone; SLID, second-line injectable drugs.

other substitutions (g1177a and 1204 ins a) were found susceptible to the three SLID. Among the 37 isolates, one isolate was $\mathrm{Km}$-resistant and did not have any mutations in the $r r s$ gene.

It is worth noting that $3 / 3(100 \%) \mathrm{XDR}$ isolates and $12 / 16$ (75\%) pre-XDR isolates carried mutations in the pncA gene conferring resistance to PZA. In addition, one of the three (33.3\%) XDR isolates, referred to as "XDR+" in this study, was totally resistant to all 4 FLD (INH, RIF, EMB and STM) and 4 SLD (Ofx, Am, Km and $\mathrm{Cm}$ ) tested, as well as resistant to PZA by carrying mutation T49P in the pncA gene.

\section{Genotyping}

Genotypic analysis was performed on the 25 isolates phenotypically resistant to at least one SLD and for which molecular data and pDST were in agreement. Spoligotype patterns of 24 isolates were found in the international database (SITVIT2) and one unique pattern (4\%) was declared "orphan". Four families were identified among the 24 isolates, including Beijing, EAI2-nonthaburi/EAI5, Manuancestor and Zero-copy families. The Beijing family was the most predominant, representing $79.2 \%(\mathrm{n}=19)$, followed by EAI $(8 \%, n=2)$, Manu ancestor $(8 \%, n=2)$ and Zero-copy $(4 \%, n=1)$. Seventeen isolates shared the Beijing-SIT1 spoligotype pattern, accounting for $70.8 \%$ of all isolates in the study (see Table S4). When comparing spoligotyping results with drug resistance patterns, the predominant Beijing family was found in $78.3 \%(18 / 23), 80.0 \%(4 / 5), 75.0 \%(12 / 16)$ and $100 \%(3 / 3)$ of Ofx-resistant, SLID-resistant, pre-XDR and XDR M.tb isolates, respectively (see Table 3). Manuancestor and Zero-copy isolates were found in $12.5 \%$ (2/ 16) and $8.3 \%(1 / 16)$ of pre-XDR isolates, respectively, while the 2 isolates of the EAI- $2 / 5$ family were found in nonMDR isolates. 

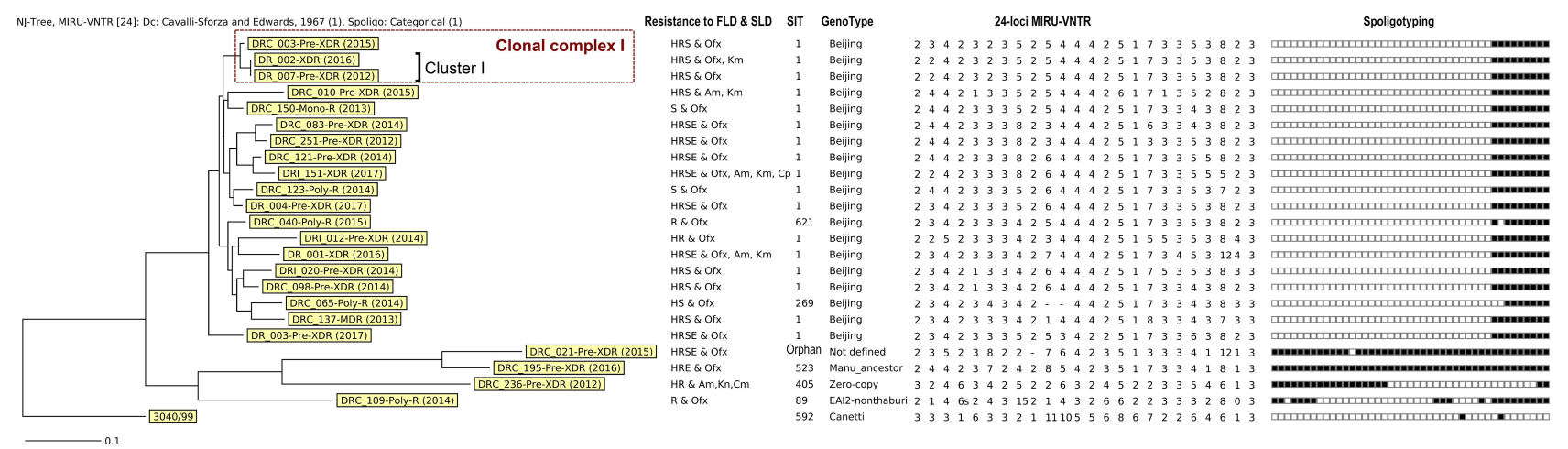

Figure 2 Phylogenetic analysis of spoligotyping and 24 loci MIRU-VNTR results of 23 Mycobacterium tuberculosis isolates resistant to SLD. Abbreviations: Am, amikacin; Cm, capreomycin; Canetti: Mycobacterium canetti used as Outgroup; E, ethambutol; FLD, first-line drugs; $\mathrm{H}$, isoniazid; Km, kanamycin; Ofx, ofloxacin; R, rifampicin; S, streptomycin; SLD, second-line drugs.

Among the 25 isolates, MIRU-VNTR analysis showed that $21(72.6 \%)$ isolates had unique MIRU-VNTR genotypes, and two $(21 \%)$ of the isolates shared the same genotype. Two isolates showed mixed MIRU-VNTR genotypes (double alleles in one locus) and were excluded from the analysis. The 24 MIRU-VNTR loci HunterGaston Discriminatory Index reached 0.9967.

Genetic variability was analyzed using MIRU-VNTR loci among the 23 sample sets and within the Beijing group $(\mathrm{n}=19)$. The genotypic diversity of all the 23 isolates was 0.956 . The average of allele numbers per locus was 3.29, ranging from 1 to 7, and 2 loci (MIRU-20 and MIRU-39) were monomorphic. Among the 19 Beijing isolates, the genotypic diversity was 0.947 and the average of allele numbers per locus was 2.29, ranging from 1 to 5 . Eight (MIRU-02, MIRU-04, MIRU-16, MIRU-20, Mtub29, ETRB, MIRU-24 and MIRU-39) of the 24 loci were monomorphic. The genetic diversity index $\mathrm{H}_{\mathrm{s}}$ was 0.378 for the entire sample and 0.211 for Beijing isolates.

A dendrogram was constructed based on both spoligotyping and MIRU-VNTR results (Figure 2) and was used to determine clusters. A cluster is defined as a group of isolates having the same MIRU-VNTR and spoligotype profiles. We observed only one cluster (Cluster I) of 2 isolates (DR_002 and DR_007) which belonged to the Beijing family. In addition, three Beijing isolates, including the two isolates of Cluster I and DRC_003, formed a clonal complex (CC I) by sharing 23 of the 24 MIRUVNTR loci. Interestingly, except for the phenotypic resistance to $\mathrm{Km}$ for isolate DR_002 and mutation G400A of the $e m b \mathrm{~B}$ gene for isolate DRC_003, the three isolates had the same pDST (resistance to INH, RIF, STM and Ofx) and mutation patterns: $k a t \mathrm{G}_{-} \mathrm{WT}$, inhA_c-15t and S94A, $r p o \mathrm{~B} \_L 511 \mathrm{P}$ and $\mathrm{H} 526 \mathrm{Q}, r p s \mathrm{~L} \_\mathrm{L} 88 \mathrm{R}, p n c \mathrm{~A} \_\mathrm{A} 171 \mathrm{E}$,
gyrA_A90V, gyrB_WT and rrs_WT. DR_007 was isolated in 2012 from a patient in Kompong Cham province and DRC_003 and DR_002 were identified from patients in Phnom Penh, three and four years later, with an additional mutation named G406A in the embB gene and an additional resistance to $\mathrm{Km}$, respectively.

\section{Treatment Outcome of Patients with Pre-XDR and XDR Isolates}

Among the 19 pre-XDR and XDR isolates identified in our study, the treatment information were available for 16 patients including two of the three patients with XDR isolates and 14 of the 16 patients with pre-XDR isolates

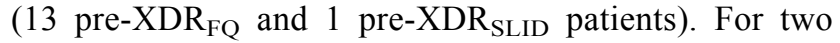
pre-XDR patients (1 pre-XDR $\mathrm{FQ}_{\mathrm{FQ}}$ and 1 pre-XDR $\left.\mathrm{SLID}_{\mathrm{S}}\right)$, the information were missing and the patient with the $\mathrm{XDR}+$ (resistant to $5 \mathrm{FLD}$ and 4 SLD) isolate refused treatment (see Table S4). The treatment information were not available for patients with SLD-resistant non-MDR isolates.

All the 16 patients initially received the 20 to 24 months standardized MDR-TB treatment regimen which is composed of a combination of FLD and SLD including SLID (Km or $\mathrm{Cm}$ ), FQ (Levofloxacin; Lfx or Moxifloxacin; Mfx), Eto, Cs, PZA and EMB. The regimen was later adjusted according to clinical evaluation and available phenotypic or molecular DST. Four patients including 2 XDR (DR_001 and DR_002) and 2 pre$\mathrm{XDR}_{\mathrm{FQ}}$ (DR_003 and DR_004) did not respond to the standardized treatment regimen and revealed FQ resistance. Thus, these four patients switched to a 24-month regimen containing Bedaquiline and SLID for 9 to 12 months as well as Cs, Clofazimine, Linezolide, PAS, PZA and EMB. 
Among the 16 patients, $12(75.0 \%)$ including 10 pre$\mathrm{XDR}_{\mathrm{FQ}}$ and $2 \mathrm{XDR}$ were cured, 1 pre-XDR $\mathrm{XDID}_{\mathrm{SLI}}(6.3 \%)$ was lost during follow-up and 3 pre-XDR $\mathrm{FQ}_{\mathrm{FQ}}(18.7 \%)$ died during the intensive phase of treatment. Two of them died after receiving the treatment for less than 1 month and one died several months after the discontinuation of the treatment due to other medical issues (see Table S4). Of the 12 patients for whom the treatment was successful, respectively, nine, two, and one had isolates belonging to the Beijing, Manu_ancestor and undefined family. The four patients who died during the treatment and the one who was lost during follow-up presented isolates belonging to the Beijing family (see Table S4). Overall, there was a $71.4 \%(10 / 14)$ and $100.0 \%(2 / 2)$ treatment success rate for pre-XDR and XDR patients, respectively.

\section{Discussion}

Although XDR TB cases have been reported worldwide, Cambodia, with nearly 35,000 cases of TB and 150 MDRTB cases annually, reported the first XDR-TB case to the WHO in $2016 .{ }^{1}$ Due to the absence of surveillance programs and systematic screening for SLD drug resistance among MDR patients, the burden of pre-XDR and XDR$\mathrm{TB}$ has been under-reported in Cambodia and is still unknown. However, in an initial previous report, the proportions of pre-XDR and XDR, based on molecular tests among MDR isolates identified between 2007 and 2009, were $14 \%$ and $1 \%$, respectively. ${ }^{38}$ Another study reported four XDR-TB cases $(0.7 \%)$ and only one pre-XDR-TB case $(0.1 \%)$ confirmed by DST among MDR-TB patients between 2006 and 2016, but only the patients who did not respond to the treatment regimen were tested for SLD resistance. ${ }^{13}$ The present study reports the first description of SLD drug resistance in M.tb in Cambodia using a combination of molecular screening for mutations in the $g y r \mathrm{~A} / \mathrm{B}$ gene conferring FQ resistance and the $r r s-\mathrm{F} 2$ gene conferring SLID resistance followed by pDST confirmation. Overall, only 25 of the 37 M.tb isolates harboring mutations in the $g y r \mathrm{~A} / \mathrm{B}$ and/or $r r s-\mathrm{F} 2$ genes were resistant to FQ or SLID by pDST. The proportion of preXDR and XDR cases among MDR isolates in our study were $13.6 \%$ and $2.5 \%$, respectively. These proportions remain lower than the global estimation of $6.2 \%$ for XDRTB and $20.8 \%$ for FQ-resistance among RR/MDR-TB but higher than the two previous reports. ${ }^{13,38}$ It should be noted that, for the first time in Cambodia, one XDR isolate (DRI_151), named XDR+ in our study, was resistant to the 4 SLD and 5 FLD tested.
Among the isolates resistant to SLD in our study, the resistance to fluoroquinolones (FQ), observed in the MDR group $(14.4 \% ; \mathrm{n}=17)$ as well as non-MDR group $(3.9 \%$, $\mathrm{n}=6$ ), was more common than the resistance to SLID. This result was expected since FQ is one of the most commonly used antibiotics in non-TB infections in Cambodia and the drug is accessible without prescription at the counter of pharmacies. ${ }^{39}$ High incidence of FQ-resistance has also been reported in many parts of the world, particularly in countries with a high TB burden. ${ }^{40}$ FQ is used in both the standardized and shorter MDR-TB regimen and is considered as an essential part of the MDR-TB regimen. Considering the high incidence of FQ-R described in our study, resistance to FQ should be determined right after patients are diagnosed with MDR-TB.

All 23 FQ-resistant isolates had mutations in the QRDR region of the gyrA gene represented by 5 SNP (see Table 2). Mutations at codon 94 were the most frequent $(69.6 \%)$, followed by codon $90(21.7 \%)$, codon 91 $(4.3 \%)$ and codon $88(4.3 \%)$. These findings are in accordance with previous reports, according to which $60.0-90 \%$ of FQ-resistant isolates had mutations at codons 88-94. ${ }^{41-43}$ The five SNP detected in our study (D94G, D94A, S91P, A90V and G88A) have also been reported to cause cross resistance among FQ compounds (Ofx, Lfx and Mfx). ${ }^{18}$ The D94G mutation was also observed in the $\mathrm{XDR}+$ isolate.

Unlike the gyrA gene, mutations in the $g y r \mathrm{~B}$ gene are less commonly associated with resistance to FQ in M.tb. ${ }^{41}$ In our study, all mutations in the gyr B gene, 7 SNP in 10 isolates, were located outside the QRDR region and most of them (10/11) were observed in FQ-susceptible isolates (see Table 2). The A644D mutation in the gyrB was only found in 1/23 FQ-resistant isolates but was observed together with the $\mathrm{D} 94 \mathrm{G}$ mutation in gyrA, known to be FQ determinant. No gyr B mutation was observed in the $\mathrm{XDR}+$ isolate. Overall, all the 9 isolates with mutations only found outside the QRDR region of gyrA/B were found susceptible to $F Q$, suggesting no impact on FQ resistance. However, one FQ-susceptible isolate harbored a double mutation in the QRDR region of the gyrA gene (see Table 2): mutation D94G, known to be FQ determinant, and mutation $\mathrm{H} 87 \mathrm{Q}$, reported in one study but not associated with FQ-resistance. ${ }^{44}$ This double gyrA mutation has never been reported elsewhere before. The presence of a double gyrA mutation in a FQsusceptible isolate might suggest: i) hetero-resistance with a lack of detection of the resistant population by the 
pDST method; ii) deactivation of FQ resistance caused by the D94G mutation due to the presence of mutation H87Q or iii) other mechanisms involved.

Resistance to SLID was found in $4.2 \%$ (5/118) of MDR isolates, three times less than the rate of FQ resistance. This can be explained by the fact that in Cambodia, injectable agents are not usually used to treat common infections compared to FQ. Mutation a1401g in the $r r s-F 2$ gene is reported as the most prominent mutation causing cross-resistance among SLID. ${ }^{20,45,46}$ This mutation was also found in the XDR + isolate conferring resistance to all the 3 SLID tested. In our study, cross-resistance was detected in 4/5 SLIDresistant isolates and 3 of them carried the a1401g mutation, in agreement with previous reports. One isolate (DR_001), carrying a new mutation named c1489t, also exhibited cross-resistance to Am and $\mathrm{Km}$. Another isolate (DR_002) resistant only to Km did not reveal any mutations in the rrs gene, suggesting the contribution of other mechanisms. It is worth noting that two isolates with mutations (g1177a and 1204 ins a) located outside the 1400-1500 region of the rrs gene were susceptible to all the SLID tested, suggesting no impact on SLID resistance.

Briefly, out of the 37 isolates harboring mutations in genes associated with SLD resistance (ie, $r r s-\mathrm{F} 2$, gyrA and gyr B), 12 were SLD susceptible by pDST. Among these 12 SLD susceptible isolates, 11 isolates carried mutations not conferring resistance and one isolate carried the D94G mutation linked to FQ resistance in association with other mutations (see above). Even if the sampling is small, these results suggest that molecular detection of SLD resistance is beneficial in our study in complement to pDST and is time-saving.

All pre-XDR and XDR isolates harbored genetic mutations conferring RIF and INH resistance. The most prevalent mutations were located in codon rpoB $\mathrm{S} 531 \mathrm{~L}$ $(52.6 \% ; 10 / 19)$ and $k a t \mathrm{G} \mathrm{S} 315 \mathrm{~T}(73.7 \% ; 14 / 19)$ in agreement with previous reports. ${ }^{43,47}$ Globally, more than $75 \%$ and $90 \%$ of the pre-XDR and XDR isolates, respectively, have been reported with phenotypic resistance to PZA. ${ }^{48}$ In our study, mutations conferring PZA resistance were observed in all XDR isolates and $70 \%$ of pre-XDR isolates. Since PZA resistance is not routinely tested in Cambodia, the drug is systematically added to the treatment regimen for drug-resistant TB patients when there is no clinical contraindication for its use. Our findings underline that the resistance to PZA should be assessed among
MDR-TB before using this drug in pre-XDR and XDR-TB treatment regimens.

Regarding the spoligotyping and MIRU-VNTR typing, the dominant genotype was Beijing, representing $81.3 \%$ of pre-XDR and $100 \%$ of XDR isolates, in agreement with previous reports in Cambodia and other parts of the world. $^{38,42,43,46}$ MIRU-VNTR profiles were heterogeneous with 22 genotypes out of 23 isolates. One cluster of two isolates belonged to the Beijing family (DR_002-XDR -2016 and DR_007-pre-XDR-2012). With another additional isolate (DRC_003-pre-XDR-2015), they form a clonal complex by sharing 23 of the 24 MIRU-VNTR loci and 8 out of 9 genetic patterns of drug resistance genes. This finding suggests that resistant isolates propagate in Cambodia and, particularly in our study, are able to evolve from pre-XDR to XDR. This underlines the absolute necessity of a better control strategy in terms of diagnostic and treatment to avoid the spread of highly resistant strains such as the XDR+ strain (DRI_151).

The treatment success rate in our study was high, $71.4 \%$ for pre-XDR TB and $100.0 \%$ for XDR-TB, compared to the WHO latest treatment outcomes of $56 \%$ for MDR-TB and 39\% for XDR-TB. However, among the pre-XDR-TB patients who received the standardized MDR-TB treatment regimen, only $57.1 \%$ (8/12) were cured. It is worth noting that the patient with the highly resistant XDR+ Beijing isolate (DRI_151) refused treatment. This XDR+ strain was isolated in 2017 from a smear-positive sputum sample from a patient who lived in Phnom Penh capital city, suggesting a high risk of transmission of this strain in the population and a possible evolution towards more antibiotic resistance. ${ }^{49}$

Since we used a limited set of genes to screen for resistance of M.tb isolates to SLD, we could not determine the resistance mechanisms for the $\mathrm{Km}$ resistance in one out of five isolates resistant to SLID. Furthermore, pDST was only performed on mutant isolates in our study. Therefore, it is likely that we underestimated the proportion of FQ- and SLID-resistant isolates for which the resistance mechanism involved other resistant genes, such as eis promotor, whi $\mathrm{B}$, tlyA or genes encoding mycobacterial efflux pumps. ${ }^{18,50}$

\section{Conclusion}

The present report is the first description of SLD resistance including pre-XDR and XDR M.tb isolates in Cambodia using both molecular and phenotypic methods. Our data suggest that the proportion of XDR and pre-XDR among MDR isolates remains low in 
Cambodia but on the rise compared to previous reports. The increasing proportion of pre-XDR and XDR M.tb isolates, the detection of a XDR + isolate and the absence of systematic susceptibility testing raise concern for the patients. Indeed, pre-XDR and XDR patients were treated as MDR-TB patients and thus received suboptimal treatment, which is likely to contribute to poor patient outcomes, DR resistant strain transmission and the acquisition of additional drug resistances. Taking this current evidence into consideration, PZA should not be used in the treatment regimen for preXDR TB and XDR-TB unless the resistance has been ruled out. DST using phenotypic or molecular methods for PZA should be performed for all MDR-TB patients in order to avoid a treatment failure and the risk of transmission of highly resistant strains. The genotypic results confirmed that pre-XDR Beijing strains circulate in the population and can evolve into highly resistant forms of XDR isolates. These results indicate the need for rapid interventions to prevent the spread of these highly resistant isolates and the emergence of extremely resistant isolates. Further study should be performed prospectively to better understand the current burden of SLD resistance in M.tb in Cambodia and the mechanisms responsible for XDR and $\mathrm{XDR}+$ resistance.

\section{Abbreviations}

Am, amikacin; CC, clonal complex; Cm, capreomycin; Cs, Cycloserine; DNA, deoxyribonucleic acid; DR, Drugresistant; DST, drug susceptibility testing; EAI, East AfricanIndian; EMB, ethambutol; Eto, Ethionamide; FLD, first-line drugs; FQ, fluoroquinolones; HGDI, Hunter-GastonDiscriminatory Index; HIV, human immunodeficiency virus; INH, isoniazid; Km, kanamycin; Lfx, Levofloxacin; LJ, Löwenstein-Jensen; M.tb, Mycobacterium tuberculosis; MDR, multidrug-resistant; Mfx, Moxifloxacin; NTP, National Tuberculosis Program; Ofx, ofloxacin; PCR, polymerase chain reaction; pDST, phenotypic drug susceptibility testing; Pre-XDR, pre-extensively drug-resistant; PSA, P-aminosalicylic acid; PZA, pyrazinamide; QDR, Quadruple drug resistance; QRDR, quinolone resistancedetermining region; $R$, resistance; RIF, rifampin; RR, RIF resistant; RRDR, rifampicin resistance-determining region; S, Susceptible; SIT, spoligotype international type; SLD, second-line drugs; SLID, second-line injectable drugs; SNP, single-nucleotide polymorphism; STM, streptomycin; TB, tuberculosis; WHO, World Health Organization; $\mathrm{XDR}$, extensively drug-resistant.

\section{Data Sharing Statement}

The data supporting the findings of this study are available within the article and the supplementary data file.

\section{Ethics Statement}

This study protocol was approved by the National Ethics Committee for Health Research, Phnom Penh, Cambodia ( $\mathrm{N}^{\mathrm{o}} 191$ NECHR). Informed consent was not sought from each patient due to the retrospective nature of the study and only M.tb isolates that were collected from patients for diagnosis or follow-up purposes were used. The study is endorsed by the Cambodian NTP before starting data collection and all the patient data was anonymized or maintained with confidentiality. This study was conducted in accordance with the Declaration of Helsinki.

\section{Acknowledgments}

We are very grateful to the patients who participated in the study. We sincerely thank the Cambodian National TB Program (CENAT) as well as their partners which support the MDR-TB program such as MSF and CHC, for providing samples or MDR TB isolates as well as their time and effort in data collection. We would like to thank the Institut Pasteur du Cambodge, IRD, CNRS and the University of Montpellier for their technical support. We thank Heidi Lançon for the English revision of the manuscript.

\section{Funding}

This study was funded by the project of the International Joint Laboratory on Drug Resistance in South-East Asia (LMI DRISA), the French Embassy in Cambodia, the Cambodian Ministry of Education, Youth and Sports (MoEYS) and the PHC Lotus project "Application of DNA chip technology for the development of diagnostic kits for rapid detection of drug-resistant tuberculosis in Vietnam, Laos and Cambodia (2014-2018)" granted by the Ministry of Science and Technology (MOST) and the French Embassy, Vietnam. Sokleaph Cheng, PhD, was funded by the IRD PhD Grant program, ARTS.

\section{Disclosure}

The authors declare no conflicts of interest for this work and that there is no conflict of interest regarding the publication of this paper. 


\section{References}

1. WHO. World Health Organization Global Tuberculosis Report 2018. Geneva: Switzerland; 2018.

2. WHO. Drug-resistant TB: totally drug-resistant TB FAQ; 2019. Available from: https://www.who.int/tb/areas-of-work/drug-resistanttb/totally-drug-resistant-tb-faq/en/. Accessed October 2, 2019.

3. Velayati AA, Masjedi MR, Farnia P, et al. Emergence of new forms of totally drug-resistant tuberculosis bacilli: super extensively drug-resistant tuberculosis or totally drug-resistant strains in Iran. Chest. 2009;136(2):420-425. doi:10.1378/chest.08-2427

4. Udwadia ZF, Amale RA, Ajbani KK, Rodrigues C. Totally drug-resistant tuberculosis in India. Clin Infect Dis. 2012;54 (4):579-581. doi:10.1093/cid/cir889

5. Banerjee R, Allen J, Westenhouse J, et al. Extensively drug-resistant tuberculosis in california, 1993-2006. Clin Infect Dis. 2008;47 (4):450-457. doi:10.1086/590009

6. Pinto L, Menzies D. Treatment of drug-resistant tuberculosis. Infect Drug Resist. 2011;4:129-135. doi:10.2147/IDR.S10332

7. WHO. World Health Organization Technical Report on Critical Concentrations for Drug Susceptibility Testing of Medicines Used in the Treatment of Drug-Resistant Tuberculosis. Geneva: Switzerland; 2018.

8. WHO. World Health Organization consolidated guidelines on drugresistant tuberculosis treatment. Geneva: Switzerland; 2019.

9. WHO. World Health Organization Guidelines for Treatment of DrugSusceptible Tuberculosis and Patient Care, 2017 Update. Geneva: Switzerland; 2017.

10. CENAT. Tuberculosis Standard Treatment Regimens. Phnom Penh, Cambodia: National Center for Tuberculosis and Leprosy Control, Ministry of Health; 2011.

11. WHO. World Health Organization Treatment of Tuberculosis Guidelines. Fourth ed. Geneva: Switzerland; 2010.

12. Migliori GB, De Iaco G, Besozzi G, Centis R, Cirillo DM. First tuberculosis cases in Italy resistant to all tested drugs. Euro Surveill. 2007;12(5):E070517.070511. doi:10.2807/esw.12.20.03194-en

13. Sam S, Shapiro AE, Sok T, et al. Initiation, scale-up and outcomes of the Cambodian National MDR-TB programme 2006-2016: hospital and community-based treatment through an NGO-NTP partnership. BMJ Open Respir Res. 2018;5(1):e000256-e000256. doi:10.1136/ bmjresp-2017-000256

14. CENAT. Guideline for Tuberculosis Control. National Center for Tuberculosis and Leprosy Control, Ministry of Health; 2016.

15. WHO. World Health Organization Treatment Guidelines for DrugResistant Tuberculosis, 2016 Update. Geneva: Switzerland; 2016.

16. Garrigo M, Aragon LM, Alcaide F, et al. Multicenter laboratory evaluation of the $\mathrm{MB} / \mathrm{BacT}$ Mycobacterium detection system and the BACTEC MGIT 960 system in comparison with the BACTEC 460TB system for susceptibility testing of Mycobacterium tuberculosis J Clin Microbiol. 2007;45(6):1766-1770. doi:10.1128/ JCM.02162-06

17. WHO. World Health Organization Updated Interim Critical Concentrations for First-Line and Second-Line DST. Geneva: Switzerland; 2012.

18. Miotto P, Tessema B, Tagliani E, et al. A standardised method for interpreting the association between mutations and phenotypic drug resistance in Mycobacterium tuberculosis. Eur Respir J. 2017;50:6.

19. Hall TA. BioEdit: a user-friendly biological sequence alignment editor and analysis program for Windows 95/98/NT. In Nucleic acids symposium series; 1999; 41: 95-98. [London]: Information Retrieval Ltd., c1979-c2000.

20. WHO. World Health Organization the Use of Next-Generation Sequencing Technologies for the Detection of Mutations Associated with Drug Resistance in Mycobacterium tuberculosis Complex: Technical Guide. Geneva: Switzerland; 2018.
21. Camus JC, Pryor MJ, Medigue C, Cole ST. Re-annotation of the genome sequence of Mycobacterium tuberculosis H37Rv. Microbiology. 2002;148 (Pt 10):2967-2973. doi:10.1099/00221287-148-10-2967

22. Walker TM, Kohl TA, Omar SV, et al. Whole-genome sequencing for prediction of Mycobacterium tuberculosis drug susceptibility and resistance: a retrospective cohort study. Lancet Infect Dis. 2015;15 (10):1193-1202. doi:10.1016/S1473-3099(15)00062-6

23. Allix-Beguec C, Arandjelovic I, Bi L, et al. Prediction of Susceptibility to First-Line Tuberculosis Drugs by DNA Sequencing. N Engl J Med. 2018;379(15):1403-1415.

24. Ramirez-Busby SM, Valafar F. Systematic review of mutations in pyrazinamidase associated with pyrazinamide resistance in Mycobacterium tuberculosis clinical isolates. Antimicrob Agents Chemother. 2015;59(9):5267-5277. doi:10.1128/AAC.00204-15

25. Kamerbeek J, Schouls L, Kolk A, et al. Simultaneous detection and strain differentiation of Mycobacterium tuberculosis for diagnosis and epidemiology. $J$ Clin Microbiol. 1997;35(4):907-914. doi:10.1128/JCM.35.4.907-914.1997

26. Filliol I, Driscoll JR, van Soolingen D, et al. Snapshot of moving and expanding clones of Mycobacterium tuberculosis and their global distribution assessed by spoligotyping in an international study. $J$ Clin Microbiol. 2003;41(5):1963-1970. doi:10.1128/JCM.41.5.19631970.2003

27. Couvin D, David A, Zozio T, Rastogi N. Macro-geographical specificities of the prevailing tuberculosis epidemic as seen through SITVIT2, an updated version of the Mycobacterium tuberculosis genotyping database. Infect Genet Evol. 2018.

28. Vitol I, Driscoll J, Kreiswirth B, Kurepina N, Bennett KP. Identifying Mycobacterium tuberculosis complex strain families using spoligotypes. Infect Genet Evol. 2006;6(6):491-504. doi:10.1016/j. meegid.2006.03.003

29. Supply P, Allix C, Lesjean S, et al. Proposal for standardization of optimized mycobacterial interspersed repetitive unit-variable-number tandem repeat typing of Mycobacterium tuberculosis. $J$ Clin Microbiol. 2006;44(12):4498-4510. doi:10.1128/JCM.01392-06

30. Hunter PR. Reproducibility and indices of discriminatory power of microbial typing methods. J Clin Microbiol. 1990;28(9):1903-1905. doi:10.1128/JCM.28.9.1903-1905.1990

31. Mokrousov I. Revisiting the Hunter Gaston discriminatory index: note of caution and courses of change. Tuberculosis. 2017;104:20-23. doi:10.1016/j.tube.2017.02.002

32. Nei M, Chesser RK. Estimation of fixation indices and gene diversities. Ann Hum Genet. 1983;47(3):253-259. doi:10.1111/ j.1469-1809.1983.tb00993.x

33. Cavalli-Sforza LL, Edwards AWF. Phylogenetic analysis: models and estimation procedures. Evolution. 1967;21(3):550-570. doi:10.1111/ j.1558-5646.1967.tb03411.x

34. Allix-Beguec C, Harmsen D, Weniger T, Supply P, Niemann S. Evaluation and strategy for use of MIRU-VNTRplus, a multifunctional database for online analysis of genotyping data and phylogenetic identification of Mycobacterium tuberculosis complex isolates. J Clin Microbiol. 2008;46(8):2692-2699. doi:10.1128/ JCM.00540-08

35. Ramaswamy SV, Reich R, Dou SJ, et al. Single nucleotide polymorphisms in genes associated with isoniazid resistance in Mycobacterium tuberculosis. Antimicrob Agents Chemother. 2003;47(4):1241-1250. doi:10.1128/AAC.47.4.1241-1250.2003

36. Rigouts L, Gumusboga M, de Rijk WB, et al. Rifampin resistance missed in automated liquid culture system for Mycobacterium tuberculosis isolates with specific rpoB mutations. J Clin Microbiol. 2013;51(8):2641-2645. doi:10.1128/JCM.02741-12

37. Al-Mutairi NM, Ahmad S, Mokaddas E, Eldeen HS, Joseph S. Occurrence of disputed rpoB mutations among Mycobacterium tuberculosis isolates phenotypically susceptible to rifampicin in a country with a low incidence of multidrug-resistant tuberculosis. BMC Infect Dis. 2019;19(1):3. doi:10.1186/s12879-018-3638-z 
38. Surcouf C, Heng S, Pierre-Audigier C, et al. Molecular detection of fluoroquinolone-resistance in multi-drug resistant tuberculosis in Cambodia suggests low association with XDR phenotypes. BMC Infect Dis. 2011;11:255. doi:10.1186/1471-2334-11-255

39 Reed TAN, Krang S, Miliya T, et al. Antimicrobial resistance in Cambodia: a review. Int J Infect Dis. 2019;85:98-107. doi:10.1016/ j.ijid.2019.05.036

40. Jabeen K, Shakoor S, Hasan R. Fluoroquinolone-resistant tuberculosis: implications in settings with weak healthcare systems. Int J Infect Dis. 2015;32:118-123. doi:10.1016/j.ijid.2015.01.006

41. Avalos E, Catanzaro D, Catanzaro A, et al. Frequency and geographic distribution of gyrA and gyrB mutations associated with fluoroquinolone resistance in clinical Mycobacterium tuberculosis isolates: a systematic review. PLoS One. 2015;10(3):e0120470. doi:10.1371/ journal.pone.0120470

42. Duong DA, Nguyen THD, Nguyen TNL, et al. Beijing genotype of Mycobacterium tuberculosis is significantly associated with high-level fluoroquinolone resistance in Vietnam. Antimicrob Agents Chemother. 2009;53(11):4835-4839. doi:10.1128/AAC.00541-09

43. Mokrousov I, Otten T, Manicheva O, et al. Molecular characterization of ofloxacin-resistant Mycobacterium tuberculosis strains from Russia. Antimicrob Agents Chemother. 2008;52(8):2937-2939. doi:10.1128/AAC.00036-08

44. Blanco D, Perez-Herran E, Cacho M, et al. Mycobacterium tuberculosis gyrase inhibitors as a new class of antitubercular drugs. Antimicrob Agents Chemother. 2015;59(4):1868-1875. doi:10.1128/ AAC.03913-14
45. Sowajassatakul A, Prammananan T, Chaiprasert A, Phunpruch S. Molecular characterization of amikacin, kanamycin and capreomycin resistance in $\mathrm{M} / \mathrm{XDR}-\mathrm{TB}$ strains isolated in Thailand. $B M C$ Microbiol. 2014;14:165. doi:10.1186/1471-2180-14-165

46. Ei PW, Aung WW, Nyunt WW, et al. Extensively drug-resistant tuberculosis in Myanmar: burden and mutations causing second-line drug resistance. Int $J$ Tuberc Lung Dis. 2018;22(1):47-53. doi:10.5588/ijtld.17.0321

47. Oudghiri A, Karimi H, Chetioui F, et al. Molecular characterization of mutations associated with resistance to second-line tuberculosis drug among multidrug-resistant tuberculosis patients from high prevalence tuberculosis city in Morocco. BMC Infect Dis. 2018;18 (1):98. doi:10.1186/s12879-018-3009-9

48. Günther $\mathrm{G}$, van Leth $\mathrm{F}$, Altet $\mathrm{N}$, et al. Beyond multidrug-resistant tuberculosis in Europe: a TBNET study. Int J Tuberc Lung Dis. 2015;19(12):1524-1527. doi:10.5588/ijtld.15.0274

49. Nguyen QH, Contamin L, Nguyen TVA, Bañuls A-L. Insights into the processes that drive the evolution of drug resistance in Mycobacterium tuberculosis. Evol Appl. 2018;11(9):1498-1511. doi:10.1111/eva.12654

50. Gygli SM, Borrell S, Trauner A, Gagneux S. Antimicrobial resistance in Mycobacterium tuberculosis: mechanistic and evolutionary perspectives. FEMS Microbiol Rev. 2017;41(3):354-373. doi:10.1093/femsre/fux011
Infection and Drug Resistance

\section{Publish your work in this journal}

Infection and Drug Resistance is an international, peer-reviewed openaccess journal that focuses on the optimal treatment of infection (bacterial, fungal and viral) and the development and institution of preventive strategies to minimize the development and spread of resistance. The journal is specifically concerned with the epidemiology of

\section{Dovepress}

antibiotic resistance and the mechanisms of resistance development and diffusion in both hospitals and the community. The manuscript management system is completely online and includes a very quick and fair peerreview system, which is all easy to use. Visit http://www.dovepress.com/ testimonials.php to read real quotes from published authors. 